\title{
THE VALUE OF INSIDER INFORMATION FOR SUPER-REPLICATION WITH QUADRATIC TRANSACTION COSTS
}

\author{
YAN DOLINSKY* AND JONATHAN ZOUARI \\ HEBREW UNIVERSITY
}

\begin{abstract}
We study super-replication of European contingent claims in an illiquid market with insider information. Illiquidity is captured by quadratic transaction costs and insider information is modeled by an investor who can peek into the future. Our main result describes the scaling limit of the superreplication prices when the number of trading periods increases to infinity. Moreover, the scaling limit gives us the asymptotic value of being an insider.
\end{abstract}

\section{INTRODUCTION}

This paper deals with super-replication of European options with insider information and quadratic transaction costs. We model insider information by assuming that the investor is allowed to peek $\epsilon$ into the future. This can be viewed as a progressive enlargement of filtrations. Although the impact of insider information on utility maximization was largely studied (see, for instance [1, 2, 3, 21, 24]), to the best of our knowledge the impact of insider information on super-replication was not studied at all.

Our modeling of illiquidity via quadratic transaction costs follows the approach of Çetin, Jarrow and Protter (see [11]) who model illiquidity via supply curves. Supply curves correspond to the case where the size of the trade has an immediate but temporary effect on the price of the asset. A linear impact results in quadratic transaction costs. For more details, see the survey paper [20.

In setups with quadratic transaction costs, continuous-time models are known to produce no liquidity effect at all, see [5] and [11. The intuition behind this result is that due to density arguments the investor can restrict himself to continuous and bounded variation trading strategies, and for this type of strategies the quadratic transaction costs are zero. Hence, in the continuous time models, a prophet investor can super-replicate a claim with arbitrary small amount. Namely, the super-hedging price is minus infinity.

In order to avoid the above "unreasonable" setup, we consider the discretetime version of the quadratic transaction costs model. Our sole assumption on the price dynamics is that the absolute value of the returns is bounded both from below and above. This can be viewed as the discrete-time Bachelier version of the

Date: October 1, 2020.

2010 Mathematics Subject Classification. 91G10, 91G20, 60F05.

Key words and phrases. Insider trading, Scaling limits, Super-replication, Quadratic costs, Volatility uncertainty.

${ }^{*}$ Corresponding author. 
widely studied uncertain volatility models, (see, e.g., [15, 22, 23, 25, 28]). We study the scaling limit of the corresponding super-replication prices when the number of trading periods goes to infinity and the time step goes to zero (continuous-time limit). Moreover, we assume that the future time interval that the investor can predict tends to zero linearly in the time step.

Our main result (Theorem 2.5) is the characterization of the scaling limit as a stochastic volatility control problem defined on the Wiener space. The main result provides a non-trivial conclusion (Corollary 2.7) which gives the asymptotic value of insider information.

We divide the proof of Theorem 2.5 into two main steps, namely proving the lower bound (Proposition 2.2) and proving the upper bound (Proposition 2.4).

For the lower bound, we identify the correct dual representation for the superreplication prices, and study the limit of the dual terms and its dependence on the insider information.

For the second step, namely, the upper bound, our main idea is to identify, in the presence of insider information, a "good" subclass of trading strategies. Good subclass is understood in the sense that from one hand it does not change the asymptotic behavior of the super-replication prices and on the other hand gives a comfortable dual representation, which allows to obtain tightness of the corresponding consistent price systems. This idea can be seen as the extension of the technique recently developed in [6], for the setup of insider trading and model uncertainty.

Our main result is not only a generalization of Theorem 2.7 in [7] for the setup of insider information, but also an improvement of this theorem. In [7, in order to get tightness of the consistent price systems (needed for the proof of the upper bound), one had to impose linear growth constraints on transaction costs and only allowed quadratic costs in an even smaller region around zero. In this paper we overcome this technical assumption by identifying a "good" subclass of constrained trading strategies. So Theorem 2.5 contains a contribution for the "usual" (i.e. no insider information) case as well.

A natural extension of the current work is to consider the more realistic setup where the insider trading strategy has a permanent impact on the stock prices. Intuitively, if we consider an investor who can pick only small time $\epsilon$ into the future, such price impact will result in a prohibitively costly super-replication prices. One way to overcome this is to follow [6] and consider scaling limits where the permanent price impact tends to zero. However, as we showed in [6] for the setup without insider information, the asymptotic behaviour of such models is the same as models with quadratic transaction costs. Hence, a more interesting and challenging direction will be to combine the present methodology of asymptotic analysis together with the Kyle-Back equilibrium modeling of insider trading (see [4, 9, 10, 13]). We leave this type of extension for future research.

The rest of the paper is organized as follows. In the next section we introduce the setup and formulate the main results. In Section 3 we prove the lower bound. In Section 4 we prove the upper bound. 


\section{Preliminaries and Main Results}

Let $0<\underline{\sigma} \leq \bar{\sigma}<\infty$ be two given constants which represent the volatility bounds. Consider the sample space

$$
\Omega:=\left\{\omega=\left(x_{1}, x_{2}, \ldots\right) \in \mathbb{R}^{\mathbb{N}}: \underline{\sigma} \leq\left|x_{i}\right| \leq \bar{\sigma}, \forall i \in \mathbb{N}\right\}
$$

with the Borel $\sigma$-algebra and the canonical process

$$
X_{k}(\omega):=x_{k} \text { for } \omega=\left(x_{1}, x_{2}, \ldots\right) .
$$

For any $n \in \mathbb{N}$ consider a financial market with a riskless savings account and a risky stock. The savings account will be used as a numeraire and thus we normalize its value to $\equiv 1$. The stock price evolution $S^{n}=\left\{S_{k}^{n}\right\}_{k=0}^{n}$ is given by a Bachelier's type of model

$$
S_{k}^{n}:=s+\frac{1}{\sqrt{n}} \sum_{i=1}^{k} X_{i}, \quad k=0,1, \ldots, n
$$

where $s \in \mathbb{R}$ is a constant.

We do not impose a particular probabilistic model and our sole assumption on the stock price dynamics is that the absolute values of the increments lie in the interval $[\underline{\sigma} / \sqrt{n}, \bar{\sigma} / \sqrt{n}]$.

We fix $N \in \mathbb{N}$ and consider an investor who can peek $N$ trading periods into the future. Namely, the filtration of the investor is given by $\mathcal{G}_{k}:=\sigma\left\{X_{1}, \ldots, X_{k+N}\right\}$, $k \in \mathbb{Z}_{+}$.

Next, let $\Lambda>0$ be a constant and consider a quadratic cost function $\beta \rightarrow \Lambda \beta^{2}$ where $\beta$ is the number of traded stocks. Thus, for the $n$-step model a trading strategy is a map $\gamma:\{0,1, \ldots, n-1\} \times \Omega \rightarrow \mathbb{R}$ such that $\gamma_{k}:=\gamma(k, \omega)$ is $\mathcal{G}_{k}$ measurable function which is specifying the number of shares held at any period $k=0,1 \ldots, n-1$.

The evolution of the mark-to-market value $\left\{Y_{k}^{\gamma}\right\}_{k=0}^{n}$ resulting from a trading strategy $\gamma$ is given by

$$
Y_{k}^{\gamma}:=\sum_{i=0}^{k-1}\left(\gamma_{i}\left(S_{i+1}^{n}-S_{i}^{n}\right)-\Lambda\left(\gamma_{i}-\gamma_{i-1}\right)^{2}\right), \quad k=0,1, \ldots, n
$$

where we set $\gamma_{-1} \equiv 0$. Hence, we start with zero stocks in our portfolio and trading to the new position $\gamma_{i}$ to be held after time $i$ incurs the transaction costs $\Lambda\left(\gamma_{i}-\gamma_{i-1}\right)^{2}$ which is the only friction in our model. The value $Y_{k}^{\gamma}$ represents the portfolios mark-to-market value at time $k$. Note that, focussing on the mark-tomarket value rather than the liquidation value, we disregard in particular the costs of unwinding any non-zero position for simplicity.

Remark 2.1. In order to make the analysis less technical we assume that $\underline{\sigma}>0$, this can be viewed as assuming that the market is sufficiently volatile. In this case the stock fluctuation (which might be positive or negative) satisfies

$$
\left|S_{k+N}^{n}-S_{k}^{n}\right| \geq \frac{\underline{\sigma} N}{n}>0, \quad k=0,1, \ldots, n-N .
$$

Hence, the investor can use his insider information in order to create an arbitrage opportunities. However, due to quadratic transaction costs the volume of the trade needs to be bounded. Thus, although the investor has an arbitrage opportunity, the super-replication problem still makes sense and the super-replication price is larger than $-\infty$. 
The main result of this paper is a scaling limit theorem for the super-replication costs, letting the number of trading periods $n$ over the time span $[0,1]$ tend to infinity while re-scaling the time between trades as $1 / n$.

Let $D[0,1]$ be the space of all RCLL (right continuous with left limits) functions $p=\left(p_{t}\right)_{t \in[0,1]}:[0,1] \rightarrow \mathbb{R}$. Let $H: D[0,1] \rightarrow \mathbb{R}$ be a continuous map with respect to the Skorohod metric

$$
d(p, q):=\inf _{\chi}\left\{\sup _{0 \leq t \leq 1}|t-\chi(t)|+\sup _{0 \leq t \leq 1}\left|p_{t}-q_{\chi(t)}\right|\right\}, \forall p, q \in D[0,1]
$$

where the infimum is over all strictly increasing continuous time changes $\chi:[0,1] \rightarrow$ $[0,1]$ with $\chi(0)=0$ and $\chi(1)=1$.

For any $n \in \mathbb{N}$ consider a European option in the $n$-step model with the payoff $Z_{n}:=H\left(\left\{S_{[n t]}^{n}\right\}_{t=0}^{1}\right)$ where [.] denotes the integer part of $\cdot$. The process $\left\{S_{[n t]}^{n}\right\}_{t=0}^{1}$ is viewed as a measurable map from $\Omega$ to $D[0,1]$. The super-replication price $\pi_{n}\left(Z_{n}\right)$ is then defined as

$$
\pi_{n}\left(Z_{n}\right):=\inf \left\{y \in \mathbb{R}: \exists \gamma \text { with } y+Y_{n}^{\gamma}(\omega) \geq Z_{n}(\omega) \forall \omega \in \Omega\right\} .
$$

We emphasize that we require the construction of a robust super-replication strategy which leads to a terminal value that dominates the payoff in any conceivable scenario $\omega \in \Omega$.

Before we arrive at the main results we need some preparations. Let $\Gamma$ be the set of all bounded (uniformly in time and space), nonnegative progressively measurable processes $\nu=\left\{\nu_{t}\right\}_{t=0}^{1}$ on the Wiener space $\left(\Omega^{W}, \mathcal{F}^{W},\left(\mathcal{F}_{t}^{W}\right)_{0 \leq t \leq 1}, \mathbb{P}^{W}\right)$ with Wiener process $W$ and its generated filtration $\left(\mathcal{F}_{t}^{W}\right)_{0 \leq t \leq 1}$. For any $\nu \in \Gamma$ we define the process $\left\{S_{t}^{\nu}\right\}_{t=0}^{1}$

$$
S_{t}^{\nu}:=s+\int_{0}^{t} \nu_{u} d W_{u}, \quad 0 \leq t \leq 1
$$

In addition, introduce the (deterministic) function $G: \mathbb{R}_{+} \rightarrow \mathbb{R}_{+}$

$$
G(z):= \begin{cases}\left(\underline{\sigma}-\frac{z}{\sigma}\right)^{2}, & 0 \leq z<\underline{\sigma}^{2}, \\ 0, & \underline{\sigma}^{2} \leq z \leq \bar{\sigma}^{2}, \\ \left(\frac{z}{\bar{\sigma}}-\bar{\sigma}\right)^{2}, & z>\bar{\sigma}^{2} .\end{cases}
$$

Now, we are ready to formulate our main results. We start with the formulation of the lower bound which will be proved in Section 3

Proposition 2.2. Let $H: D[0,1] \rightarrow \mathbb{R}$ be a continuous function with respect to the Skorohod metric. Moreover, assume that there exists constants $C, \mu>0$ such that

$$
|H(p)| \leq C\left(1+\sup _{0 \leq t \leq T}\left|p_{t}\right|^{\mu}\right), \quad \forall p \in D[0,1] .
$$

Then,

$$
\lim \inf _{n \rightarrow \infty} \pi_{n}\left(Z_{n}\right) \geq \sup _{\nu \in \Gamma} \mathbb{E}_{\mathbb{P} W}\left(H\left(S^{\nu}\right)-\frac{N}{4 \Lambda}\left|S_{1}^{\nu}-s\right|^{2}-\frac{1}{16 \Lambda} \int_{0}^{1} G\left(\nu_{t}^{2}\right) d t\right) .
$$

The following simple corollary will be used in the proof of the upper bound.

Corollary 2.3. Let $H: D[0,1] \rightarrow \mathbb{R}_{+}$be a continuous function which satisfies (2.2). Then,

$$
\lim \inf _{n \rightarrow \infty} \pi_{n}\left(Z_{n}\right)>-\infty
$$


Proof. Follows from choosing $\nu \equiv 0$ in the right hand side of (2.3).

Next, we formulate the upper bound which will be proved in Section 4

Proposition 2.4. Let $H: D[0,1] \rightarrow \mathbb{R}_{+}$be nonnegative and Lipschitz continuous with respect to the Skorohod metric. Then,

$$
\lim \sup _{n \rightarrow \infty} \pi_{n}\left(Z_{n}\right) \leq \sup _{\nu \in \Gamma} \mathbb{E}_{\mathbb{P} W}\left(H\left(S^{\nu}\right)-\frac{N}{4 \Lambda}\left|S_{1}^{\nu}-s\right|^{2}-\frac{1}{16 \Lambda} \int_{0}^{1} G\left(\nu_{t}^{2}\right) d t\right) .
$$

Let us notice that if $H$ is Lipschitz continuous with respect to the Skorohod metric then it satisfies the growth condition (2.2) (with $\mu=1$ ). We now combine the statements of the above propositions and state them as the main theorem of our paper.

Theorem 2.5. Let $H: D[0,1] \rightarrow \mathbb{R}_{+}$be nonnegative and Lipschitz continuous with respect to the Skorohod metric. Then,

$$
\lim _{n \rightarrow \infty} \pi_{n}\left(Z_{n}\right)=\sup _{\nu \in \Gamma} \mathbb{E}_{\mathbb{P} W}\left(H\left(S^{\nu}\right)-\frac{N}{4 \Lambda}\left|S_{1}^{\nu}-s\right|^{2}-\frac{1}{16 \Lambda} \int_{0}^{1} G\left(\nu_{t}^{2}\right) d t\right) .
$$

Proof. Follows from Proposition 2.2 and Proposition 2.4

Remark 2.6. For the case $N=0$ (no insider information) our results are improvement of Theorem 2.7 in 7 in the sense that we do not impose linear growth constraints on the transaction costs. Moreover, from Proposition 2.2 and Remark 4.8 it follows that Theorem 2.5 can be easily extended to payoffs of the from

$$
\hat{H}(p):=H(p)-\alpha\left|p_{1}-p_{0}\right|^{2}, \quad \forall p \in D[0,1]
$$

where $H$ is as above (nonnegative and Lipschitz continuous) and $\alpha>0$ is a positive constant. This observation is important for the following corollary.

Corollary 2.7. Theorem 2.5 says that asymptotically the super-replication price of the European claim $H(S)$ for an insider who can peek $N$ trading periods into the future equals to the super-replication price for an "usual" investor of the claim $H(S)-\frac{N}{4 \Lambda}\left|S_{1}-s\right|^{2}$. In other words, for super-replication with quadratic transaction costs, the asymptotic value of peeking $N$ trading periods into the future equals to holding (for zero costs) a European option with payoff $\frac{N}{4 \Lambda}\left|S_{1}-s\right|^{2}$.

We end this section with the following remark which discusses what happens if $N$ increases to infinity with $n$.

Remark 2.8. Let $H$ be as in Theorem 2.5. First we argue that

$$
\lim _{N \rightarrow \infty} \sup _{\nu \in \Gamma} \mathbb{E}_{\mathbb{P} W}\left(H\left(S^{\nu}\right)-\frac{N}{4 \Lambda}\left|S_{1}^{\nu}-s\right|^{2}-\frac{1}{16 \Lambda} \int_{0}^{1} G\left(\nu_{t}^{2}\right) d t\right)=H(\bar{s})-\frac{\underline{\sigma}^{2}}{16 \Lambda}
$$

where $\bar{s} \in D[0, T]$ is the constant function $\bar{s} \equiv s$. Indeed, by taking $\nu=0$ in the above left hand side we get

$$
\lim \inf _{N \rightarrow \infty} \sup _{\nu \in \Gamma} \mathbb{E}_{\mathbb{P} W}\left(H\left(S^{\nu}\right)-\frac{N}{4 \Lambda}\left|S_{1}^{\nu}-s\right|^{2}-\frac{1}{16 \Lambda} \int_{0}^{1} G\left(\nu_{t}^{2}\right) d t\right) \geq H(\bar{s})-\frac{\underline{\sigma}^{2}}{16 \Lambda} .
$$

On the other hand, from the Doob-Kolmogorov inequality and the Lipschitz continuity of $H$ it follows that there exists a constant $L$ such that

$$
\mathbb{E}_{\mathbb{P} W}\left(H\left(S^{\nu}\right)\right) \leq H(\bar{s})+L \mathbb{E}_{\mathbb{P} W}\left(\sup _{0 \leq t \leq T}\left|S_{t}^{\nu}-s\right|\right) \leq H(\bar{s})+2 L\left(\mathbb{E}_{\mathbb{P} W}\left(\left|S_{1}^{\nu}-s\right|^{2}\right)\right)^{1 / 2} .
$$


This, together with the Itô Isometry $\mathbb{E}_{\mathbb{P}^{W}}\left(\left|S_{1}^{\nu}-s\right|^{2}\right)=\mathbb{E}_{\mathbb{P} W}\left(\int_{0}^{1} \nu_{t}^{2} d t\right)$, and the simple inequality $G(z) \geq \underline{\sigma}^{2}-\frac{2 \bar{\sigma} z}{\underline{\sigma}}, \forall z>0$ gives

$$
\lim \sup _{N \rightarrow \infty} \sup _{\nu \in \Gamma} \mathbb{E}_{\mathbb{P} W}\left(H\left(S^{\nu}\right)-\frac{N}{4 \Lambda}\left|S_{1}^{\nu}-s\right|^{2}-\frac{1}{16 \Lambda} \int_{0}^{1} G\left(\nu_{t}^{2}\right) d t\right) \leq H(\bar{s})-\frac{\underline{\sigma}^{2}}{16 \Lambda},
$$

and (2.5) follows.

From Theorem 2.5 and (2.5) we conclude that if $N$ increases to infinity with $n$, then asymptotically the super-replication price will be less or equal than $H(\bar{s})-\frac{\frac{\sigma}{2}^{2}}{16 \Lambda}$. This value does not depend on $\bar{\sigma}$, and so can be viewed as unreasonably low. Thus, roughly speaking, the "right" scaling is to keep $N$ fixed as $n$ goes to infinity.

\section{Proof of The LOWER Bound}

In this section we prove Proposition 2.2, We start with the following lemma.

Lemma 3.1. For any $n$,

$$
\pi_{n}\left(Z_{n}\right) \geq \sup _{\mathbb{P} \in \mathcal{P}} \mathbb{E}_{\mathbb{P}}\left(Z_{n}-\frac{1}{4 \Lambda} \sum_{i=0}^{n-1}\left(\mathbb{E}_{\mathbb{P}}\left(S_{n}^{n} \mid \mathcal{G}_{i}\right)-S_{i}^{n}\right)^{2}\right)
$$

where $\mathcal{P}$ is the set of all Borel probability measures on $\Omega$.

Proof. Let $y \in \mathbb{R}$ be an initial capital and $\gamma$ be a trading strategy such that

$$
y+Y_{n}^{\gamma}(\omega) \geq Z_{n}(\omega), \quad \forall \omega \in \Omega .
$$

Then, for any $\mathbb{P} \in \mathcal{P}$

$$
\begin{gathered}
\mathbb{E}_{\mathbb{P}}\left(Z_{n}\right) \leq y+\mathbb{E}_{\mathbb{P}}\left(\sum_{i=0}^{n-1}\left(\gamma_{i}\left(S_{i+1}^{n}-S_{i}^{n}\right)-\Lambda\left(\gamma_{i}-\gamma_{i-1}\right)^{2}\right)\right) \\
=y+\mathbb{E}_{\mathbb{P}}\left(\sum_{i=0}^{n-1}\left(\left(\gamma_{i}-\gamma_{i-1}\right)\left(S_{n}^{n}-S_{i}^{n}\right)-\Lambda\left(\gamma_{i}-\gamma_{i-1}\right)^{2}\right)\right) \\
=y+\mathbb{E}_{\mathbb{P}}\left(\sum_{i=0}^{n-1}\left(\left(\gamma_{i}-\gamma_{i-1}\right)\left(\mathbb{E}_{\mathbb{P}}\left(S_{n}^{n} \mid \mathcal{G}_{i}\right)-S_{i}^{n}\right)-\Lambda\left(\gamma_{i}-\gamma_{i-1}\right)^{2}\right)\right) \\
\leq y+\mathbb{E}_{\mathbb{P}}\left(\frac{1}{4 \Lambda} \sum_{i=0}^{n-1}\left(\mathbb{E}_{\mathbb{P}}\left(S_{n}^{n} \mid \mathcal{G}_{i}^{n}\right)-S_{i}^{n}\right)^{2}\right)
\end{gathered}
$$

where the last inequality follows from the simple inequality $\beta \alpha-\Lambda \beta^{2} \leq \frac{\alpha^{2}}{4 \Lambda}, \quad \alpha, \beta \in$ $\mathbb{R}$. Thus,

$$
y \geq \mathbb{E}_{\mathbb{P}}\left(Z_{n}-\frac{1}{4 \Lambda} \sum_{i=0}^{n-1}\left(\mathbb{E}_{\mathbb{P}}\left(S_{n}^{n} \mid \mathcal{G}_{i}\right)-S_{i}^{n}\right)^{2}\right) .
$$

Since $\mathbb{P} \in \mathcal{P}$ was arbitrary we complete the proof.

Remark 3.2. In fact, there is an equality in (3.1). Namely, for the case where the payoff $Z_{n}$ is a continuous function of $S_{1}^{n}, \ldots, S_{n}^{n}$ (since $H$ is continuous, this holds true in our setup) we have

$$
\pi_{n}\left(Z_{n}\right)=\sup _{\mathbb{P} \in \mathcal{P}} \mathbb{E}_{\mathbb{P}}\left(Z_{n}-\frac{1}{4 \Lambda} \sum_{i=0}^{n-1}\left(\mathbb{E}_{\mathbb{P}}\left(S_{n}^{n} \mid \mathcal{G}_{i}\right)-S_{i}^{n}\right)^{2}\right) .
$$

The proof can be done by following the discretization technique from [7. (see Theorem 2.2 there) or the approach from [12] which is based on representation results for increasing convex functionals. We omit the proof, and provide only the lower bound (this is Lemma 3.1) which is essential for proving Proposition 2.2. 
Next, let $C[0,1]$ be the space of all continuous functions $p:[0,1] \rightarrow \mathbb{R}$ equipped with the supremum norm $\|p\|:=\sup _{0 \leq t \leq 1}\left|p_{t}\right|$.

Definition 3.3. Let $\Gamma_{0} \subset \Gamma$ be the set of all nonnegative, continuous processes $\nu=\left\{\nu_{t}\right\}_{t=0}^{1}$ defined on the Wiener space $\left(\Omega^{W}, \mathcal{F}^{W},\left(\mathcal{F}_{t}^{W}\right)_{0 \leq t \leq 1}, \mathbb{P}^{W}\right)$ which are given by

$$
\nu_{t}=f(t, W), \quad t \in[0,1]
$$

where $f:[0,1] \times C[0,1] \rightarrow \mathbb{R}_{+}$satisfies the following conditions.

(i) For any $t \in[0,1]$ and $p, q \in C[0,1]$, if $p_{[0, t]}=q_{[0, t]}$ then $f(t, p)=f(t, q)$. Namely, $f$ is a progressively measurable map.

(ii) The map $f$ is bounded, i.e. $\sup _{(t, p) \in[0,1] \times C[0,1]} f(t, p)<\infty$.

(iii) The function $f$ is bounded away from zero, i.e. $\inf _{(t, p) \in[0,1] \times C[0,1]} f(t, p)>0$.

(iv) There is $\delta=\delta(f)>0$ such that $f_{[1-\delta, 1] \times C[0,1]} \equiv \bar{\sigma}$.

(v) There exists a constant $\mathcal{L}=\mathcal{L}(f)$ such that

$$
\left|f\left(t_{1}, p\right)-f\left(t_{2}, q\right)\right| \leq \mathcal{L}\left(\left|t_{1}-t_{2}\right|+|| p-q \|\right) \quad \forall\left(t_{1}, t_{2}, p, q\right) \in[0,1]^{2} \times C^{2}[0,1] .
$$

We arrive at the following result.

Lemma 3.4. Let $\nu \in \Gamma_{0}$. There exists a sequence of Borel probability measures $\left\{\mathbb{Q}_{n}\right\}_{n \in \mathbb{N}}$ on $\Omega$ such that we have the weak convergence

$$
\left\{S_{[n t]}^{n}\right\}_{t=0}^{1} \Rightarrow\left\{S_{t}^{\nu}\right\}_{t=0}^{1} \text { on } D[0,1] \text {. }
$$

Namely, the distribution of $\left\{S_{[n t]}^{n}\right\}_{t=0}^{1}$ under $\mathbb{Q}_{n}$ converge weakly to the distribution of the process $\left\{S_{t}^{\nu}\right\}_{t=0}^{1}$ given by (2.1). Moreover,

$$
\lim _{n \rightarrow \infty} \mathbb{E}_{\mathbb{Q}_{n}}\left(\sum_{k=0}^{n-1}\left(\mathbb{E}_{\mathbb{Q}_{n}}\left(S_{n}^{n} \mid \mathcal{G}_{k}\right)-S_{k}^{n}\right)^{2}\right)=\mathbb{E}_{\mathbb{P} W}\left(N\left|S_{1}^{\nu}-s\right|^{2}+\frac{1}{4} \int_{0}^{1} G\left(\nu_{t}^{2}\right) d t\right) .
$$

Proof. For $n \in \mathbb{N}$ and a sequence $A=\left(A_{0}, \ldots, A_{n}\right) \in \mathbb{R}^{n+1}$ we denote by $\widetilde{A}$ the continuous linear interpolation in $C[0,1]$ of the points $A_{0}, \ldots, A_{n}$. Formally,

$$
\widetilde{A}_{t}:=(n t-[n t]) A_{[n t+1]}+(1+[n t]-n t) A_{[n t]}, \quad t \in[0,1] .
$$

Let $\nu_{t}=f(t, W), t \in[0,1]$ where $f$ satisfies conditions (i) $-(\mathrm{v})$ in Definition 3.3 Fix $n \in \mathbb{N}$ and define on $\Omega$ the processes $\left\{\sigma_{k}^{n}\right\}_{k=0}^{n},\left\{\kappa_{k}^{n}\right\}_{k=0}^{n},\left\{B_{k}^{n}\right\}_{k=0}^{n}$ and $\left\{\xi_{k}^{n}\right\}_{k=1}^{n}$ by the following recursion:

$$
\sigma_{0}^{n}:=\underline{\sigma} \vee f(0,0) \wedge \bar{\sigma}, \quad \kappa_{0}^{n}:=0, \quad B_{0}^{n}:=0
$$

and, for $k=1, \ldots, n$

$$
\begin{gathered}
\sigma_{k}^{n}:=\underline{\sigma} \vee f\left(\frac{k-1}{n}, \widetilde{B^{n}}\right) \wedge \bar{\sigma}, \\
\kappa_{k}^{n}:=\frac{1}{2}\left(\frac{f^{2}\left(\frac{k-1}{n}, \widetilde{B^{n}}\right)}{\left|\sigma_{k}^{n}\right|^{2}}-1\right), \\
\xi_{k}^{n}:=\frac{X_{k}}{\sigma_{k}^{n}}, \\
B_{k}^{n}:=B_{k-1}^{n}+\frac{1}{\sqrt{n}} \frac{\left(1+\kappa_{k}^{n}\right) X_{k}-\kappa_{k-1}^{n} X_{k-1}}{\sqrt{1+2 \kappa_{k}^{n}} \sigma_{k}^{n}}
\end{gathered}
$$

where recall, $X_{1}, X_{2}, \ldots$ is the canonical process on $\Omega$.

From properties (ii)-(iii) in Definition 3.3 and the assumption $\underline{\sigma}>0$ it follows that $\kappa^{n}, \frac{1}{1+2 \kappa^{n}}$ are uniformly bounded in $n$. Hence, $B_{k}^{n}-B_{k-1}^{n}=\bar{O}\left(n^{-1 / 2}\right)$ (unless 
we state otherwise, the $O$ term is uniform in space and time). Moreover, the progressive measurability of $f$ (property (i) in Definition 3.3) ensures that its valuation in the definition of $\sigma_{k}^{n}$ and $\kappa_{k}^{n}$ depends on $B^{n}$ only via its already constructed values $B_{0}^{n}, \ldots, B_{k-1}^{n}$.

Next, we observe that $\sigma_{k}^{n} \in[\underline{\sigma}, \bar{\sigma}]$ for all $k$. Thus, there exists a probability measure $\mathbb{Q}_{n}$ on the canonical space $\Omega$ (an atomic measure) such that under $\mathbb{Q}_{n}$ the random variables $\xi_{1}^{n}, \ldots, \xi_{n}^{n}= \pm 1 \mathbb{Q}_{n}$ a.s. and

$$
\mathbb{Q}_{n}\left(\xi_{k}^{n}= \pm 1 \mid \xi_{1}^{n}, \ldots, \xi_{k-1}^{n}\right):=\frac{1}{2}\left(1 \pm \frac{\kappa_{k-1}^{n} \sigma_{k-1}^{n} \xi_{k-1}^{n}}{\sigma_{k}^{n}\left(1+\kappa_{k}^{n}\right)}\right), \quad k=1, \ldots, n
$$

where we set $\xi_{0}^{n} \equiv 1$. From property (v) in Definition 3.3. the estimate $B_{k}^{n}-B_{k-1}^{n}=$ $O\left(n^{-1 / 2}\right)$ and the fact that $\sigma^{n}$ and $1+2 \kappa^{n}$ are bounded away from zero it follows that for sufficiently large $n$ the right hand side of (3.4) is in $[0,1]$. Thus, (for sufficiently large $n$ ) the probability measure $\mathbb{Q}_{n}$ is well defined.

Now consider the stochastic process $\left\{M_{k}^{n}\right\}_{k=0}^{n}$ given by

$$
M_{k}^{n}:=S_{k}^{n}+\frac{\kappa_{k}^{n} X_{k}}{\sqrt{n}}, \quad k=0, \ldots, n
$$

where we set $X_{0} \equiv \sigma_{0}^{(n)}$. Observe that (3.4) yields that $\left\{B_{k}^{n}\right\}_{k=0}^{n}$ and $\left\{M_{k}^{n}\right\}_{k=0}^{n}$ are $\mathbb{Q}_{n}$ martingales. From the definition of $\mathbb{Q}_{n}$ and the regularity properties of the function $f$ we have

$$
\begin{gathered}
\mathbb{E}_{\mathbb{Q}_{n}}\left(\left|B_{k}^{n}-B_{k-1}^{n}\right|^{2} \mid X_{1}, \ldots, X_{k-1}\right) \\
=\frac{1}{n} \frac{\left(1+\kappa_{k}^{n}\right)^{2}\left|\sigma_{k}^{n}\right|^{2}-\left|\kappa_{k-1}^{n} \sigma_{k-1}^{n}\right|^{2}}{\left(1+2 \kappa_{k}^{n}\right)\left|\sigma_{k}^{n}\right|^{2}} \\
=\frac{1}{n}+O\left(n^{-3 / 2}\right) .
\end{gathered}
$$

This together with the Martingale Central Limit theorem (see Proposition 1 in [26]) gives

$$
\left\{B_{[n t]}^{n}\right\}_{t=0}^{1} \Rightarrow W \text { on } D[0,1]
$$

Notice that

$$
M_{k}^{n}-M_{k-1}^{n}=\sqrt{1+2 \kappa_{k}^{n}} \sigma_{k}^{n}\left(B_{k}^{n}-B_{k-1}^{n}\right), \quad k=1, \ldots, n .
$$

Thus, by combining the stability result Theorem 5.4 in [16 together with (3.5), the regularity of $f$ and the simple estimate $M^{n}-S^{n}=O\left(n^{-1 / 2}\right)$ we obtain

$$
\left(M_{[n t]}^{n}, S_{[n t]}^{n}\right)_{t=0}^{1} \Rightarrow\left(S_{t}^{\nu}, S_{t}^{\nu}\right)_{t=0}^{1} \text { on } D[0,1] \times D[0,1]
$$

and (3.2) follows.

Next, we show (3.3). From property (iv) in Definition 3.3 it follows that for sufficiently large $n, S_{n}^{n}=M_{n}^{n}$. Hence,

$$
M_{k}^{n}=\mathbb{E}_{\mathbb{Q}_{n}}\left(S_{n}^{n} \mid X_{1}, \ldots, X_{k}\right), \quad k=0,1, \ldots, n .
$$


This together with the equality $\left|X_{k}\right|^{2}=\frac{\left|\sigma_{k}^{n}\right|^{2}}{n} \mathbb{Q}_{n}$ a.s., yields

$$
\begin{gathered}
\sum_{k=0}^{n-1}\left(\mathbb{E}_{\mathbb{Q}_{n}}\left(S_{n}^{n} \mid \mathcal{G}_{k}\right)-S_{k}^{n}\right)^{2} \\
=\sum_{k=0}^{n-1-N}\left(M_{k+N}^{n}-S_{k}^{n}\right)^{2}+O(1 / n) \\
=\frac{1}{n} \sum_{k=0}^{n-1-N}\left(\kappa_{k+N}^{n} X_{k+N}+\sum_{j=1}^{N} X_{k+j}\right)^{2}+O(1 / n) \\
=\frac{1}{n} \sum_{i=0}^{n-1}\left|\kappa_{i}^{n}\right|^{2}\left|\sigma_{i}^{n}\right|^{2}+\frac{N}{n} \sum_{i=0}^{n-1}\left|\sigma_{i}^{n}\right|^{2}+2 \sum_{j=1}^{N} I_{j}^{n}+O(1 / n)
\end{gathered}
$$

where

$$
I_{j}^{n}:=\frac{1}{n}\left(\sum_{k=0}^{n-1-N}\left(1+\kappa_{k+N}^{n}\right) X_{k+N} X_{k+j}+\sum_{k=0}^{n-1-N} \sum_{i=j+1}^{N-1} X_{k+i} X_{k+j}\right) .
$$

From the continuity and boundedness of $f$ we get the weak convergence (on $\mathbb{R}$ )

$$
\frac{1}{n} \sum_{i=0}^{n-1}\left|\kappa_{i}^{n}\right|^{2}\left|\sigma_{i}^{n}\right|^{2} \Rightarrow \frac{1}{4} \int_{0}^{1} G\left(\nu_{t}^{2}\right) d t
$$

and

$$
\frac{N}{n} \sum_{i=0}^{n-1}\left|\sigma_{i}^{n}\right|^{2} \Rightarrow N \int_{0}^{1}\left(\underline{\sigma} \vee \nu_{t} \wedge \bar{\sigma}\right)^{2} d t
$$

Next, we fix $j$ and estimate $I_{j}^{n}$. Writing $I_{j}^{n}$ as a telescopic sum we have

$$
\begin{gathered}
I_{j}^{n}=\frac{1}{n} \sum_{i=j+1}^{N} \sum_{k=0}^{n-1-N}\left(\left(1+\kappa_{k+i}^{n}\right) X_{k+i} X_{k+j}-\kappa_{k+i-1}^{n} X_{k+i-1} X_{k+j}\right) \\
+\frac{1}{n} \sum_{k=0}^{n-1} \kappa_{k}^{n} X_{k}^{2}+O(1 / n) \\
=\sum_{i=j+1}^{N} \sum_{k=0}^{n-1-N}\left(S_{k+j}^{n}-S_{k+j-1}^{n}\right)\left(M_{k+i}^{n}-M_{k+i-1}^{n}\right) \\
+\frac{1}{n} \sum_{k=0}^{n-1} \kappa_{k}^{n}\left|\sigma_{k}^{n}\right|^{2}+O(1 / n) .
\end{gathered}
$$

From Theorem 4.1 in 16 and (3.6) we obtain

$$
\sum_{k=0}^{n-1-N}\left(S_{k+j}^{n}-S_{k+j-1}^{n}\right)\left(M_{k+i}^{n}-M_{k+i-1}^{n}\right) \Rightarrow \int_{0}^{1}\left(S_{t}^{\nu}-S_{t}^{\nu}\right) d S_{t}^{\nu}=0, \quad i=j+1, \ldots, N .
$$

Hence, for any $j=1, \ldots, N$

$$
I_{j}^{n} \Rightarrow \frac{1}{2}\left(\int_{0}^{1} \nu_{t}^{2} d t-\int_{0}^{1}\left(\underline{\sigma} \vee \nu_{t} \wedge \bar{\sigma}\right)^{2} d t\right) .
$$

This together with (3.7)-(3.9) and the uniform boundedness of $\sigma^{n}, \kappa^{n}$ gives (3.3).

We now have all the pieces in place that we need for the completion of the proof of the lower bound.

Proof of Proposition 2.2.

First Step: In this step we prove that (recall that $\Gamma_{0} \subset \Gamma$ )

$$
\begin{aligned}
& \sup _{\nu \in \Gamma} \mathbb{E}_{\mathbb{P} W}\left(H\left(S^{\nu}\right)-\frac{N}{4 \Lambda}\left|S_{1}^{\nu}-s\right|^{2}-\frac{1}{16 \Lambda} \int_{0}^{1} G\left(\nu_{t}^{2}\right) d t\right) \\
= & \sup _{\nu \in \Gamma_{0}} \mathbb{E}_{\mathbb{P} W}\left(H\left(S^{\nu}\right)-\frac{N}{4 \Lambda}\left|S_{1}^{\nu}-s\right|^{2}-\frac{1}{16 \Lambda} \int_{0}^{1} G\left(\nu_{t}^{2}\right) d t\right) .
\end{aligned}
$$


Let $\nu \in \Gamma$. There exists a constant $c>0$ such that $\nu \leq c d t \otimes \mathbb{P}^{W}$ a.s. Using similar density arguments as in Lemma 7.3 in [18] gives that there exists a sequence $\left\{\nu^{n}\right\}_{n=1}^{\infty} \subset \Gamma_{0}$ such that $\nu_{n} \rightarrow \nu$ in probability (with respect to $d t \otimes \mathbb{P}^{W}$ ) and for any $n$ we have $\nu^{n} \leq c d t \otimes \mathbb{P}^{W}$ a.s.

From the growth condition (2.2) we obtain that the random variables

$$
H\left(S^{\nu^{n}}\right)-\frac{N}{4 \Lambda}\left|S_{1}^{\nu^{n}}-s\right|^{2}-\frac{1}{16 \Lambda} \int_{0}^{1} G\left(\left|\nu_{t}^{n}\right|^{2}\right) d t, \quad n \in \mathbb{N}
$$

are uniformly integrable, and so from the continuity of $H$

$$
\begin{gathered}
\mathbb{E}_{\mathbb{P} W}\left(H\left(S^{\nu}\right)-\frac{N}{4 \Lambda}\left|S_{1}^{\nu}-s\right|^{2}-\frac{1}{16 \Lambda} \int_{0}^{1} G\left(\nu_{t}^{2}\right) d t\right) \\
=\lim _{n \rightarrow \infty} \mathbb{E}_{\mathbb{P} W}\left(H\left(S^{\nu^{n}}\right)-\frac{N}{4 \Lambda}\left|S_{1}^{\nu^{n}}-s\right|^{2}-\frac{1}{16 \Lambda} \int_{0}^{1} G\left(\left|\nu_{t}^{n}\right|^{2}\right) d t\right)
\end{gathered}
$$

and the first step is completed.

Second Step: In view of the first step, in order to complete the proof of Proposition 2.2 it remains to show that for any $\nu \in \Gamma_{0}$

$$
\lim \inf _{n \rightarrow \infty} \pi_{n}\left(Z_{n}\right) \geq \mathbb{E}_{\mathbb{P} W}\left(H\left(S^{\nu}\right)-\frac{N}{4 \Lambda}\left|S_{1}^{\nu}-s\right|^{2}-\frac{1}{16 \Lambda} \int_{0}^{1} G\left(\nu_{t}^{2}\right) d t\right) .
$$

Choose $\nu \in \Gamma_{0}$. Consider the probability measures $\mathbb{Q}_{n}, n \in \mathbb{N}$ and the corresponding martingales $M^{n}, n \in \mathbb{N}$ which are constructed in Lemma 3.4.

Observe that $\left|M_{k}^{n}-M_{k-1}^{n}\right|=O\left(n^{-1 / 2}\right)$ for all $k \leq n$. Thus, from Lemma 3.3 in [8] it follows that (recall the constant $\mu$ from Proposition 2.2).

$$
\sup _{n \in \mathbb{N}} \mathbb{E}_{\mathbb{Q}_{n}}\left(\max _{0 \leq k \leq n}\left|M_{k}^{n}\right|^{2 \mu}\right)<\infty \text {. }
$$

From (2.2) and the simple bound $\left|S^{n}-M^{n}\right|=O\left(n^{-1 / 2}\right)$ we obtain that $H\left(\left\{S_{[n t]}^{n}\right\}_{t=0}^{1}\right)$, $n \in \mathbb{N}$ are uniformly integrable. This together with (3.2) and the continuity of $H$ gives

$$
\lim _{n \rightarrow \infty} \mathbb{E}_{\mathbb{Q}_{n}}\left(H\left(\left\{S_{[n t]}^{n}\right\}_{t=0}^{1}\right)\right)=\mathbb{E}_{\mathbb{P} W}\left(H\left(S^{\nu}\right)\right) .
$$

Finally, from Lemma 3.1, (3.3) and (3.10) it follows that

$$
\begin{gathered}
\liminf \inf _{n \rightarrow \infty} \pi_{n}\left(Z_{n}\right) \\
\geq \liminf \mathbb{E}_{\mathbb{Q}_{n}}\left(H\left(\left\{S_{[n t]}^{n}\right\}_{t=0}^{1}\right)-\frac{1}{4 \Lambda} \sum_{i=0}^{n-1}\left(\mathbb{E}_{\mathbb{P}}\left(S_{n}^{n} \mid \mathcal{G}_{i}\right)-S_{i}^{n}\right)^{2}\right) \\
=\mathbb{E}_{\mathbb{P} W}\left(H\left(S^{\nu}\right)-\frac{N}{4 \Lambda}\left|S_{1}^{\nu}-s\right|^{2}-\frac{1}{16 \Lambda} \int_{0}^{1} G\left(\nu_{t}^{2}\right) d t\right)
\end{gathered}
$$

and the proof is completed.

\section{Proof of the UPPER Bound}

In this section we prove Proposition 2.4

We start with a discretization of the set $\Omega$. For any $k \in \mathbb{N}$ let $\Omega^{k}$ be the set of all sequences $\left(x_{1}, x_{2}, \ldots\right) \in \Omega$ such that for any $i \in \mathbb{N}$ we have

$$
\left|x_{i}\right|=\frac{j}{k} \underline{\sigma}+\left(1-\frac{j}{k}\right) \bar{\sigma}
$$

for some $j=j(i) \in\{0,1, \ldots, k\}$. 
For any $k, n \in \mathbb{N}$ consider the $n$-step financial market supported on the set $\Omega^{k} \subset \Omega$. Observe that this is a (finite) multinomial model. For any map $Z: \Omega^{k} \rightarrow \mathbb{R}$ we define the corresponding super-replication price

$$
\pi_{n}^{k}(Z)=\inf \left\{y \in \mathbb{R}: \exists \gamma \text { with } y+Y_{n}^{\gamma}(\omega) \geq Z(\omega) \forall \omega \in \Omega^{k}\right\}
$$

where the definition of the trading strategy $\gamma$ and the corresponding wealth process $Y^{\gamma}$ are the same as in Section 2 .

Lemma 4.1. For any $n \in \mathbb{N}$,

$$
\lim _{k \rightarrow \infty} \pi_{n}^{k}\left(Z_{n}\right)=\pi_{n}\left(Z_{n}\right) .
$$

Proof. Fix $n$. Clearly $\pi_{n}^{k}\left(Z_{n}\right) \leq \pi_{n}\left(Z_{n}\right)$ for all $k$. Thus, we need to show that $\liminf _{k \rightarrow \infty} \pi_{n}^{k}\left(Z_{n}\right) \geq \pi_{n}\left(Z_{n}\right)$. Since $H: D[0,1] \rightarrow \mathbb{R}$ is continuous then there exists a continuous function $F_{n}: \mathbb{R}^{n} \rightarrow \mathbb{R}_{+}$such that $Z_{n}=F_{n}\left(S_{1}^{n}, \ldots, S_{n}^{n}\right)$.

Hence, we can mimic the proof of Lemma 3.3 in [7. The only difference is that, since in the present setup we have insider information, we need to provide a different argument to the fact that without loss of generality we can assume that for any $k$ the corresponding trading strategy $\gamma^{k}$ is uniformly bounded in $k$.

To that end choose $k \in \mathbb{N}$. Let $y$ be an initial capital and $\gamma^{k}$ be a trading strategy such that

$$
y+Y_{n}^{\gamma^{k}}(\omega) \geq Z_{n}(\omega), \quad \forall \omega \in \Omega^{k} .
$$

Since $H: D[0,1] \rightarrow \mathbb{R}$ is continuous then $\left\|Z_{n}\right\|_{\infty}:=\sup _{\omega \in \Omega} Z_{n}(\omega)<\infty$. Thus, without loss of generality we assume that $y \leq\left\|Z_{n}\right\|_{\infty}$. This together with (4.1) and the fact that $Z_{n} \geq 0$ gives

$$
\left\|Z_{n}\right\|_{\infty}+\sum_{i=0}^{n-1}\left(\frac{\bar{\sigma}}{\sqrt{n}}\left|\gamma_{i}^{k}\right|-\Lambda\left(\gamma_{i}^{k}-\gamma_{i-1}^{k}\right)^{2}\right) \geq 0, \quad \forall \omega \in \Omega^{k}
$$

and so (recall that $\gamma_{-1}^{k} \equiv 0$ ),

$$
\sup _{k \in \mathbb{N}} \max _{0 \leq i \leq n-1} \sup _{\omega \in \Omega^{k}}\left|\gamma^{k}(i, \omega)\right|<\infty
$$

as required.

From Lemma 4.1 it follows that for any $n \in \mathbb{N}$ there exists $k=k(n)$ such that $\pi_{n}^{k(n)}\left(Z_{n}\right)>\pi_{n}\left(Z_{n}\right)-\frac{1}{n}$. For simplicity we denote $\Omega_{n}:=\Omega_{n}^{k(n)}$ and $\hat{\pi}_{n}(\cdot):=\pi_{n}^{k(n)}$, i.e.

$$
\hat{\pi}_{n}\left(Z_{n}\right)>\pi_{n}\left(Z_{n}\right)-\frac{1}{n}
$$

Thus, in order to prove Proposition 2.4 we need to establish the inequality

$$
\lim \sup _{n \rightarrow \infty} \hat{\pi}_{n}\left(Z_{n}\right) \leq \sup _{\nu \in \Gamma} \mathbb{E}_{\mathbb{P} W}\left(H\left(S^{\nu}\right)-\frac{N}{4 \Lambda}\left|S_{1}^{\nu}-s\right|^{2}-\frac{1}{16 \Lambda} \int_{0}^{1} G\left(\nu_{t}^{2}\right) d t\right) .
$$

The fact that the super-replication price $\hat{\pi}_{n}(\cdot)$ is defined in the multinomial model setup will be used in our duality analysis in Proposition 4.6.

Our preliminary step in the asymptotic analysis of the super-replication prices $\hat{\pi}_{n}\left(Z_{n}\right), n \in \mathbb{N}$ is the space-time discretizations of the price processes. Specifically, 
for any $\epsilon \in(0,1)$ and $n \in \mathbb{N}$ define (on $\Omega_{n}$ ) a sequence of stopping times by the following recursive relations: $\tau_{0}^{n, \epsilon}:=0$ and for $k \in \mathbb{N}$

$$
\tau_{k}^{n, \epsilon}:=1 \wedge \inf \left\{t \geq \tau_{k-1}^{n, \epsilon}:\left|S_{[n t]}^{n}-S_{\left[n \tau_{k-1}^{n, \epsilon}\right]}\right| \geq \epsilon \text { or }\left|t-\tau_{k-1}^{n, \epsilon}\right| \geq \epsilon^{2}\right\} .
$$

Set,

$$
S_{t}^{n, \epsilon}:=\sum_{k \in \mathbb{N}} S_{\left[n \tau_{k-1}^{n, \epsilon}\right]}^{n} \mathbb{I}_{t \in\left[\tau_{k-1}^{n, \epsilon}, \tau_{k}^{n, \epsilon}\right)}+S_{n}^{n} \mathbb{I}_{t=1}, \quad 0 \leq t \leq 1
$$

As usual II denotes the indicator function.

Observe that since $H$ is Lipschitz in the Skorohod metric then $H(p)$ has a linear growth in ||$p-p_{0}||:=\sup _{0 \leq t \leq T}\left|p_{t}-p_{0}\right|$. Thus, for any $\lambda>0$ there exists $c(\lambda)>0$ such that

$$
H(p) \leq c(\lambda)+\lambda^{2}\left\|p-p_{0}\right\|^{2}, \quad \forall p \in D[0,1] .
$$

Without loss of generality we assume that $c(\lambda)>2$. Set,

$$
\begin{gathered}
K=K(\epsilon, \lambda):=\left[c(\lambda) /(\epsilon \lambda)^{2}\right]+1 \\
Z^{n, \epsilon, K}:=H\left(S^{n, \epsilon}\right) \mathbb{I}_{\left\{\tau_{K}^{n, \epsilon}=1\right\}} .
\end{gathered}
$$

Lemma 4.2. There exists $\lambda_{0} \in(0,1)$ such that for any $\epsilon \in(0,1)$ and $\lambda<\lambda_{0}$,

$$
\lim \sup _{n \rightarrow \infty}\left(\hat{\pi}_{n}\left(Z_{n}\right)-(1-\lambda) \hat{\pi}_{n}\left(Z^{n, \epsilon, K} /(1-\lambda)\right)\right) \leq O(\epsilon+\lambda) .
$$

Proof. Choose $\epsilon, \lambda \in(0,1)$. From the fact that $H$ is Lipschitz in the Skorohod metric we obtain that for sufficiently large $n$

$$
Z_{n} \leq H\left(S^{n, \epsilon}\right)+O(\epsilon) .
$$

Next, choose $n \in \mathbb{N}$ sufficiently large such that (4.6) holds true. Set,

$$
Q^{n, \epsilon}:=\sup _{0 \leq t \leq 1}\left|S_{t}^{n, \epsilon}-s\right|^{2}+\sum_{k \in \mathbb{N}}\left(\left|S_{\left[n \tau_{k}^{n, \epsilon}\right]}^{n}-S_{\left[n \tau_{k-1}^{n, \epsilon}\right]}^{n}\right|^{2}+\left|\tau_{k}^{n, \epsilon}-\tau_{k-1}^{n, \epsilon}\right|\right) .
$$

From the definition of $\tau_{k}^{n, \epsilon}, k \in \mathbb{N}$ it follows that

$$
K \epsilon^{2} \leq \sum_{k=1}^{K}\left(\left|S_{\tau_{k}^{n, \epsilon}}^{n}-S_{\tau_{k-1}^{n, \epsilon}}^{n}\right|^{2}+\left|\tau_{k}^{n, \epsilon}-\tau_{k-1}^{n, \epsilon}\right|\right) \text { on the event }\left\{\tau_{K}^{n, \epsilon}<1\right\} .
$$

This together with (4.5)-(4.6) gives

$$
Z_{n} \leq Z^{n, \epsilon, K}+\lambda^{2} Q^{n, \epsilon}+O(\epsilon)
$$

Convexity of the wealth dynamics implies convexity of the super-replication price. Hence,

$$
\hat{\pi}_{n}\left(Z_{n}\right) \leq(1-\lambda) \hat{\pi}_{n}\left(Z^{n, \epsilon, K} /(1-\lambda)\right)+\lambda \hat{\pi}_{n}\left(\lambda Q^{n, \epsilon}\right)+O(\epsilon)
$$

Following the arguments of Lemma 3.6 in [6] (replace $\sigma$ with $\bar{\sigma}$ ) we get that there exists $\lambda_{0}>0$ such that for any $\lambda<\lambda_{0}$ we have $\hat{\pi}_{n}\left(\lambda Q^{n, \epsilon}\right) \leq \lambda\left(1+36 \bar{\sigma}^{2}\right)$. This together with (4.8) completes the proof.

Remark 4.3. Although Lemma 3.6 in [6] deals with binomial models, it is straightforward to check that the same arguments will work in our setup, if we replace $\sigma$ with $\bar{\sigma}$. Clearly, insider information can not increase the super-replication price. 
The main step in the proof of the upper bound is to understand how to superreplicate the claims $Z^{n, \epsilon, K} /(1-\lambda)$ in the presence of insider information. Notice that these claims depend on the values of their underlying at only a fixed number $K$ of sampling times. Such claims turn out to allow for a particularly convenient duality estimate for their super-replication prices. The idea is that rather than looking on all trading strategies for a cost-effective super-hedge, we will consider a suitably constraint class.

Fix $n \in \mathbb{N}$ and $\epsilon>0$. Let $\left[S^{n}\right]_{k}:=\frac{1}{n} \sum_{i=1}^{k} X_{i}^{2}, k=0,1 \ldots, n$ be the quadratic variation of $S^{n}$ and let $\left(\mathcal{F}_{k}^{n, \epsilon}\right)_{k \in \mathbb{Z}_{+}}$be the filtration given by

$$
\mathcal{F}_{k}^{n, \epsilon}:=\sigma\left\{\tau_{1}^{n, \epsilon}, \ldots, \tau_{k}^{n, \epsilon}, S_{\left[n \tau_{1}^{n, \epsilon]}\right.}^{n}, \ldots, S_{\left[n \tau_{k}^{n, \epsilon}\right]}^{n},\left[S^{n}\right]_{\left[n \tau_{1}^{n, \epsilon}\right]}, \ldots,\left[S^{n}\right]_{\left[n \tau_{k}^{n, \epsilon}\right]}\right\} .
$$

We have the following computational result.

Lemma 4.4. Let $m \in \mathbb{N}$. Denote by $\mathcal{A}_{m}$ the set of all $\left(\mathcal{F}_{k}^{n, \epsilon}\right)_{k=0, \ldots, m}$-adapted processes $\left\{\alpha_{k}\right\}_{k=0}^{m}$ such that $\left|\alpha_{k}\right| \leq \log n$ for $k=0, \ldots, m$. Then, for any $\phi, \psi \in \mathcal{A}_{m}$ there exists a trading strategy $\gamma$ (in the sense that given in Section 2) such that

$$
\begin{gathered}
Y_{n}^{\gamma} \geq \sum_{k=0}^{m} \phi_{k}\left(S_{\left[n \tau_{k+1}^{n, \epsilon}\right]}^{n}-S_{\left[n \tau_{k}^{n, \epsilon}\right]}^{n}\right) \\
+\sum_{k=0}^{m}\left(\frac{\psi_{k}}{2}+\frac{N}{4 \lambda}\right)\left(S_{\left[n \tau_{k+1}^{n, \epsilon}\right]}^{n}-S_{\left[n \tau_{k}^{n, \epsilon}\right]}^{n}\right)^{2} \\
-\sum_{k=0}^{m}\left(\frac{\psi_{k}}{2}+\Lambda \psi_{k}^{2}\right)\left(\left[S^{n}\right]_{\left[n \tau_{k+1}^{n, \epsilon}\right]}-\left[S^{n}\right]_{\left[n \tau_{k}^{n, \epsilon}\right]}\right)-O\left(\log ^{2} n / n^{1 / 6}\right)
\end{gathered}
$$

The above $O$ term may depend on $\epsilon$ and $m$.

Proof. For $k=0,1, \ldots, m+1$ introduce the random variables (integer valued)

$$
a_{k}:=\left[n \tau_{k}^{n, \epsilon}\right], \quad b_{k}:=a_{k}+\left[n^{1 / 3}\right] .
$$

For $k=0,1, \ldots, m$ set $c_{k}:=a_{k+1}-\left[n^{1 / 3}\right]$. Let us define the trading strategy $\gamma$ on each of the time intervals $\left[a_{k}, a_{k+1}-1\right], k=0,1, \ldots, m$. If $a_{k}>n-2 n^{1 / 3}$ we do not trade in the time interval $\left[a_{k}, a_{k+1}-1\right]$. Otherwise (observe that for sufficiently large $n b_{k}<c_{k}$ ), we divide the interval

$$
\left[a_{k}, a_{k+1}-1\right]=\left[a_{k}, b_{k}\right) \cup\left[b_{k}, c_{k}\right) \cup\left[c_{k}, a_{k+1}-1\right)
$$

into 3 disjoint intervals. On the first interval $\left[a_{k}, b_{k}\right)$ we trade in a constant speed and change the number of shares from 0 to $\phi_{k}$. On the second interval $\left[b_{k}, c_{k}\right)$ we trade such that for any $i \in\left[b_{k}, c_{k}\right)$ we buy

$$
\psi_{k}\left(S_{i+N}^{n}-S_{i+N-1}^{n}\right)+\frac{S_{i+N}^{n}-S_{i}^{n}}{2 \Lambda}=\frac{1}{\sqrt{n}}\left(\psi_{k} X_{i+N}+\frac{\sum_{j=i+1}^{i+N} X_{j}}{2 \Lambda}\right)
$$

number of shares. Namely,

$$
\gamma_{i}-\gamma_{i-1}=\frac{1}{\sqrt{n}}\left(\psi_{k} X_{i+N}+\frac{\sum_{j=i+1}^{i+N} X_{j}}{2 \Lambda}\right), \quad i \in\left[b_{k}, c_{k}\right) .
$$

Recall, that $N$ is the number of steps that the investor can peek into the future. Finally, on the last interval $\left[c_{k}, a_{k+1}-1\right)$ we liquidate our portfolio in a constant speed.

Let us estimate the portfolio wealth of such strategy. Observe that $S^{n}$ is uniformly bounded (in $n$ ) on the time interval $\left[0, a_{m}\right]$, and so, from (4.9) we conclude that $\gamma$ is of size $O(\log n)$. Thus, the transaction costs on the (small) time 
intervals $\left[a_{k}, b_{k}\right)$ and $\left[b_{k}, c_{k}\right)$ are of order $O\left(\log ^{2} n / n^{1 / 3}\right)$. Next, we notice that on the intervals $\left[a_{k}, b_{k}\right)$ and $\left[c_{k}, a_{k+1}-1\right)$ the stock price fluctuation is of order $O\left(n^{1 / 3} n^{-1 / 2}\right)=O\left(n^{-1 / 6}\right)$. Therefore, by the summation by parts formula, (also recall the non trading time interval $\left[n-2 n^{1 / 3}, n\right]$ )

$$
\begin{gathered}
Y_{n}^{\gamma}=\sum_{k=0}^{m} \sum_{i=b_{k}}^{c_{k}-1} \gamma_{i}\left(S_{i+1}^{n}-S_{i}^{n}\right)-\Lambda\left(\gamma_{i}-\gamma_{i-1}\right)^{2}-O\left(\log ^{2} n / n^{1 / 6}\right) \\
=\sum_{k=0}^{m}\left[J_{k}^{1}-J_{k}^{2}-J_{k}^{3}\right]-O\left(\log ^{2} n / n^{1 / 6}\right)
\end{gathered}
$$

where

$$
\begin{gathered}
J_{k}^{1}:=\gamma_{c_{k}}\left(S_{c_{k}}^{n}-S_{b_{k}}^{n}\right), \\
J_{k}^{2}:=\sum_{i=b_{k}+1}^{c_{k}}\left(\gamma_{i}-\gamma_{i-1}\right)\left(S_{i}^{n}-S_{b_{k}}^{n}\right), \\
J_{k}^{3}:=\sum_{i=b_{k}}^{c_{k}-1} \Lambda\left(\gamma_{i}-\gamma_{i-1}\right)^{2} .
\end{gathered}
$$

From (4.9), the fact that $S^{n}$ is uniformly bounded on $\left[0, a_{m}\right]$ and the stock price fluctuation on the small intervals is of order $O\left(n^{-1 / 6}\right)$ we get

$$
\begin{gathered}
J_{k}^{1}=\phi_{k}\left(S_{a_{k+1}}^{n}-S_{a_{k}}^{n}\right)+\left(\psi_{k}+\frac{N}{2 \lambda}\right)\left(S_{a_{k+1}}^{n}-S_{a_{k}}^{n}\right)^{2}+O\left(\log n / n^{1 / 6}\right) \\
(4.11)=\phi_{k}\left(S_{a_{k+1}}^{n}-S_{a_{k}}^{n}\right)+\left(\frac{\psi_{k}}{2}+\frac{N}{4 \Lambda}\right)\left(\left(S_{a_{k+1}}^{n}-S_{a_{k}}^{n}\right)^{2}+\left[S^{n}\right]_{a_{k+1}}-\left[S^{n}\right]_{a_{k}}\right) \\
+\frac{1}{n}\left(\psi_{k}+\frac{N}{2 \lambda}\right) \sum_{a_{k} \leq i<j \leq a_{k+1}} X_{i} X_{j}+O\left(\log n / n^{1 / 6}\right) .
\end{gathered}
$$

Next, applying (4.9) again we obtain

$$
\begin{gathered}
J_{k}^{2}=\frac{\psi_{k}}{n} \sum_{a_{k} \leq i, j \leq a_{k+1}, j-i \geq N} X_{i} X_{j}+ \\
\frac{1}{2 \Lambda n} \sum_{p=1}^{N} \sum_{a_{k} \leq i, j \leq a_{k+1}, j-i \geq p} X_{i} X_{j}+O\left(\log n / n^{1 / 6}\right) \\
=\frac{1}{n}\left(\psi_{k}+\frac{N}{2 \lambda}\right) \sum_{a_{k} \leq i<j \leq a_{k+1}} X_{i} X_{j} \\
-\frac{1}{n} \sum_{p=1}^{N-1}\left(\psi_{k}+\frac{N-p}{2 \Lambda}\right) \sum_{a_{k} \leq i \leq a_{k+1}} X_{i} X_{i+p}+O\left(\log n / n^{1 / 6}\right)
\end{gathered}
$$

and

$$
\begin{gathered}
J_{k}^{3}=\left(\psi_{k}+\Lambda \psi_{k}^{2}+\frac{N}{4 \Lambda}\right)\left(\left[S^{n}\right]_{a_{k+1}}-\left[S^{n}\right]_{a_{k}}\right) \\
+\frac{1}{n} \sum_{p=1}^{N-1}\left(\psi_{k}+\frac{N-p}{2 \Lambda}\right) \sum_{a_{k} \leq i \leq a_{k+1}} X_{i} X_{i+p}+O\left(\log ^{2} n / n^{1 / 6}\right) .
\end{gathered}
$$

The result follows by combining (4.10)-(4.13).

Remark 4.5. Lemma 4.4 provides some intuition about the term $\frac{N}{4 \Lambda}\left|S_{1}^{\nu}-s_{0}\right|^{2}$ which appears in Theorem 2.5. Indeed, due to the insider information, the seller can "make money" from the stock price fluctuation. The corresponding term (in the formulation of Lemma 4.4) is given by

$$
\frac{N}{4 \lambda} \sum_{k=0}^{m}\left(S_{\left[n \tau_{k+1}^{n, \epsilon]}\right.}^{n}-S_{\left[n \tau_{k}^{n, \epsilon]}\right.}^{n}\right)^{2}
$$

and obviously it is closely related to quadratic variation. As we prove rigorously below, due to the Ito Isometry, the asymptotic behavior of these terms is given by $\frac{N}{4 \Lambda}\left|S_{1}^{\nu}-s_{0}\right|^{2}$.

The main idea in the proof of Lemma 4.4 is to apply trading strategies which are of the form (4.9). We obtained this type of strategies by the "guess and verify" method. Namely, by applying these strategies, we prove below that the upper bound 
for the asymptotic behaviour of the super-replication prices is equal to the lower bound of the super-replication prices which was established in Section 3.

A natural question which we leave for future research is the extension of Theorem 2.5 to transaction costs beyond quadratic. Let us notice that different types of transaction costs require different types of scaling.

To get a convenient upper bound for $\hat{\pi}_{n}\left(Z^{n, \epsilon, K} /(1-\lambda)\right)$ it will be useful to consider processes on a slightly expanded time horizon, namely on $[0,1+\lambda]$ rather than $[0,1]$. For that purpose we extend the function $H$ to $H_{1+\lambda}: D[0,1+\lambda] \rightarrow \mathbb{R}_{+}$ simply by letting, for $p \in D[0,1+\lambda]$,

$$
H_{1+\lambda}(p):=H\left([0,1] \ni t \mapsto p_{t(1+\lambda)}\right) .
$$

Fix $\epsilon, \lambda \in(0,1)$ and let $K=K(\epsilon, \lambda)$ as before. Let $\mathcal{D}^{\epsilon, \lambda}$ be the set of stochastic processes $D=\left\{D_{t}\right\}_{t=0}^{1+\lambda}$ defined on some filtered probability space $\left(\Omega^{D}, \mathcal{G}^{D},\left\{\mathcal{G}_{t}^{D}\right\}_{t=0}^{1+\lambda}, \mathbb{P}^{D}\right)$ (we assume that the filtration is right continuous and completed), have the form

$$
D_{t}=\sum_{k=1}^{K} \Upsilon_{k-1} \mathbb{I}_{t \in\left[\theta_{k-1}, \theta_{k}\right)}+\left(\Upsilon_{K}+\sigma W_{t-\theta_{K}}\right) \mathbb{I}_{t \in\left[\theta_{K}, 1+\lambda\right]}
$$

where $\theta_{k}, k=0,1 \ldots, K$ are stopping times (with respect to $\left\{\mathcal{G}_{t}^{D}\right\}_{t=0}^{1+\lambda}$ ) and

$$
\begin{gathered}
\Upsilon_{0}=s_{0}, \quad\left|\Upsilon_{k}-\Upsilon_{k-1}\right| \leq 2 \epsilon, \\
\theta_{0}=0, \quad \frac{\lambda}{K} \leq \theta_{k}-\theta_{k-1} \leq \frac{\lambda}{K}+\epsilon^{2} \\
\Upsilon_{k-1}=\mathbb{E}_{\mathbb{P} D}\left(\Upsilon_{k} \mid \mathcal{G}_{\theta_{k-1}}^{D}\right) .
\end{gathered}
$$

Moreover, $W$ is a Brownian motion independent of $\mathcal{G}_{\theta_{K}}^{D}$. As usual $\left\{[D]_{t}\right\}_{t=0}^{1+\lambda}$ denotes the quadratic variation of $D$ which is given by

$$
[D]_{t}:=\sum_{k=1}^{K} \sum_{i=1}^{k-1}\left(\Upsilon_{i}-\Upsilon_{i-1}\right)^{2} \mathbb{I}_{t \in\left[\theta_{k-1}, \theta_{k}\right)}+\left(\sum_{i=1}^{K}\left(\Upsilon_{i}-\Upsilon_{i-1}\right)^{2}+\bar{\sigma}^{2}\left(t-\theta_{K}\right)\right) \mathbb{I}_{t \in\left[\theta_{K}, 1+\lambda\right]}
$$

We also define the process $\left\{\zeta_{t}^{D}\right\}_{t=0}^{1+\lambda}$

$$
\zeta_{t}^{D}:=\sum_{k=1}^{K} \frac{\mathbb{E}_{\mathbb{P} D}\left(\Upsilon_{k}^{2}-\Upsilon_{k-1}^{2} \mid \mathcal{G}_{\theta_{k-1}}^{D}\right)}{\mathbb{E}_{\mathbb{P} D}\left(\theta_{k}-\theta_{k-1} \mid \mathcal{G}_{\theta_{k-1}}^{D}\right)} \mathbb{I}_{t \in\left[\theta_{k-1}, \theta_{k}\right)}+\bar{\sigma}^{2} \mathbb{I}_{t \in\left[\theta_{K}, 1+\lambda\right]}
$$

We remark that the filtration $\mathcal{G}^{D}$ can be larger than the usual filtration generated by $D$.

Proposition 4.6. We have the following upper bound

$$
\begin{gathered}
\lim \sup _{n \rightarrow \infty} \hat{\pi}_{n}\left(Z^{n, \epsilon, K} /(1-\lambda)\right) \leq O(\epsilon+\lambda) \\
+\sup _{D \in \mathcal{D}^{\epsilon, \lambda}} \mathbb{E}_{\mathbb{P}^{D}}\left(\frac{H_{1+\lambda}(D)}{1-\lambda}-\frac{N}{4 \Lambda}\left(\frac{1}{\lambda^{2}} \wedge[D]_{1+\lambda}\right)-\frac{1}{16 \Lambda} \int_{0}^{1+\lambda} G\left(\zeta_{t}^{D}\right) d t\right) .
\end{gathered}
$$

Proof. Choose $n \in \mathbb{N}$. Recall the set $\Omega_{n}$ defined after Lemma 4.1, the filtration $\left(\mathcal{F}_{k}^{n, \epsilon}\right)_{k \in \mathbb{Z}_{+}}$introduced before Lemma 4.4 and the set $\mathcal{A}_{m}$ introduced in Lemma 4.4. We take $m=K-1$ and denote $\mathcal{A}:=\mathcal{A}_{K-1}$.

Denote by $\hat{\mathcal{P}}_{n}$ the set of all probability measures on $\left(\Omega_{n}, \sigma\left\{X_{1}, \ldots, X_{n}\right\}\right)$. Introduce the function $\Gamma: \hat{\mathcal{P}}_{n} \times \mathcal{A} \rightarrow \mathbb{R}$ by

$$
\Gamma(\mathbb{P}, \psi):=\mathbb{E}_{\mathbb{P}}\left(Z^{\psi}-\ln n \sum_{k=0}^{K-1}\left|\mathbb{E}_{\mathbb{P}}\left(S_{\left[n \tau_{k+1}^{n, \epsilon}\right]}^{n}-S_{\left[n \tau_{k}^{n, \epsilon}\right]}^{n} \mid \mathcal{F}_{k}^{n, \epsilon}\right)\right|\right)
$$


where

$$
\begin{aligned}
Z^{\psi} & :=\frac{Z^{n, \epsilon, K}}{1-\lambda}-\sum_{k=0}^{K-1}\left(\frac{\psi_{k}}{2}+\frac{N}{4 \Lambda}\right)\left(S_{\left[n \tau_{k+1}^{n, \epsilon}\right]}^{n}-S_{\left[n \tau_{k}^{n, \epsilon}\right]}^{n}\right)^{2} \\
& +\sum_{k=0}^{K-1}\left(\frac{\psi_{k}}{2}+\Lambda \psi_{k}^{2}\right)\left(\left[S^{n}\right]_{\left[n \tau_{k+1}^{n, \epsilon}\right]}-\left[S^{n}\right]_{\left[n \tau_{k}^{n, \epsilon}\right]}\right) .
\end{aligned}
$$

From Lemma 4.4 (for $m=K-1$ ) we obtain that for sufficiently large $n$

$$
\begin{aligned}
& \hat{\pi}_{n}\left(Z^{n, \epsilon, K} /(1-\lambda)\right) \\
& <\epsilon+\inf _{\psi \in \mathcal{A}} \inf \left\{y: \exists \phi \in \mathcal{A}: y+\sum_{k=0}^{K-1} \phi_{k}\left(S_{\left[n \tau_{k+1}^{n, \epsilon}\right]}^{n}-S_{\left[n \tau_{k}^{n, \epsilon]}\right.}^{n}\right) \geq Z^{\psi}\right\} \\
& =\epsilon+\inf _{\psi \in \mathcal{A}} \sup _{\mathbb{P} \in \hat{\mathcal{P}}_{n}} \Gamma(\mathbb{P}, \psi)
\end{aligned}
$$

where the last equality follows from classical linear super-replication duality with convexly constrained strategy sets (cf. [19, Theorem 4.1 in connection with Example 2.3). Let us notice that since the probability space $\left(\Omega_{n}, \sigma\left\{X_{1}, \ldots, X_{n}\right\}\right)$ is finite, then we can define a probability measure which gives to every event a positive weight. Hence, pathwise super-replication is equivalent to almost surely superreplication, and so, we can apply the result from [19].

Next, we argue that we can switch the above inf and sup. It is readily checked that for any $\mathbb{P} \in \hat{\mathcal{P}}_{n}$ the map $\psi \rightarrow \Gamma(\psi, \mathbb{P})$ is convex and that the map $\mathbb{P} \rightarrow \Gamma(\psi, \mathbb{P})$ is concave for any $\psi$ fixed. Observing that the sets $\hat{\mathcal{P}}_{n}, \mathcal{A}$ can easily be identified with convex and compact subsets in Euclidean space, we can thus invoke the Minimax Theorem (e.g. Theorem 45.8 in [27]) to obtain

$$
\inf _{\psi \in \mathcal{A}} \sup _{\mathbb{P} \in \hat{\mathcal{P}}_{n}} \Gamma(\mathbb{P}, \psi)=\sup _{\mathbb{P} \in \hat{\mathcal{P}}_{n}} \inf _{\psi \in \mathcal{A}} \Gamma(\mathbb{P}, \psi) .
$$

From (4.19) we conclude that (for sufficiently large $n$ ) there exists a probability measure $\mathbb{P}_{n} \in \hat{\mathcal{P}}_{n}$ for which

$$
\hat{\pi}_{n}\left(Z^{n, \epsilon, K} /(1-\lambda)\right)<\epsilon+\inf _{\psi \in \mathcal{A}} \Gamma\left(\mathbb{P}_{n}, \psi\right) .
$$

By combining Corollary 2.3. (4.2) and Lemma4.2 we get $\liminf _{n \rightarrow \infty} \hat{\pi}_{n}\left(Z^{n, \epsilon, K}\right)>$ $-\infty$. On the other hand, it is straight forward to see that $\sup _{n \in \mathbb{N}}\left\|Z^{n, \epsilon, K}\right\|_{\infty}<\infty$. These observations together with (4.20) for $\psi \equiv 0$ give that for sufficiently large $n$

$$
\mathbb{E}_{\mathbb{P}_{n}}\left(\sum_{k=0}^{K-1}\left|\mathbb{E}_{\mathbb{P}_{n}}\left(S_{\left[n \tau_{k+1}^{n, \epsilon}\right]}^{n}-S_{\left[n \tau_{k}^{n, \epsilon]}\right.}^{n} \mid \mathcal{F}_{k}^{n, \epsilon}\right)\right|\right) \leq \frac{1}{\sqrt{\ln n}}
$$

Next, for $k=0,1, \ldots, K$ set

$$
\begin{gathered}
\theta_{k}:=\tau_{k}^{n, \epsilon}+\frac{\lambda}{K} k, \\
\Upsilon_{k}:=S_{\left[n \tau_{k}^{n, \epsilon]}\right]}^{n}-\sum_{i=0}^{k-1} \mathbb{E}_{\mathbb{P}_{n}}\left(S_{\left[n \tau_{i+1}^{n+\epsilon}\right]}^{n}-S_{\left[n \tau_{i}^{n, \epsilon]}\right]}^{n} \mid \mathcal{F}_{i}^{n, \epsilon}\right)
\end{gathered}
$$

and introduce the stochastic processes

$$
\begin{gathered}
\mathcal{N}_{t}=\sum_{k=1}^{K} \mathbb{I}_{t \geq \theta_{k}}, \quad 0 \leq t \leq 1+\lambda \\
U_{t}=\sum_{k=1}^{K}\left[S^{n}\right]_{\left[n \tau_{k-1}^{n, \epsilon}\right]} \mathbb{I}_{t \in\left[\theta_{k-1}, \theta_{k}\right)}+\left[S^{n}\right]_{\left[n \tau_{K}^{n, \epsilon}\right]} \mathbb{I}_{t \geq \theta_{K}}, \quad 0 \leq t \leq 1+\lambda .
\end{gathered}
$$

Let $\left\{D_{t}\right\}_{t=0}^{1+\lambda}$ be given by (4.14) and let $\mathcal{G}_{t}^{D}$ be the filtration generated by the processes $\left\{D_{t}\right\}_{t=0}^{1+\lambda},\left\{\mathcal{N}_{t}\right\}_{t=0}^{1+\lambda},\left\{U_{t}\right\}_{t=0}^{1+\lambda}$ and $\left\{W_{t \vee \theta_{K}-\theta_{K}}\right\}_{t=0}^{1+\lambda}$ where $W$ is a standard Brownian motion independent of $S^{n}$. 
Let us verify that (4.15)-4.17) hold true. Indeed, from the definitions we have $\tau_{k+1}^{n, \epsilon}-\tau_{k}^{n, \epsilon} \leq \epsilon^{2}$ and (for sufficiently large $n$ ) $\left|S_{\left[n \tau_{i+1}^{n, \epsilon}\right]}^{n}-S_{\left[n \tau_{i}^{n, \epsilon}\right]}^{n}\right| \leq 2 \epsilon$, this gives (4.15) and (4.16) respectively. The equality (4.17) follows from the simple observation that $\mathcal{G}_{\theta_{k}}^{D}=\mathcal{F}_{k}^{n, \epsilon}, k=0,1 \ldots, K$.

We wish to apply (4.20) and estimate $\inf _{\psi \in \mathcal{A}} \Gamma\left(\mathbb{P}_{n}, \psi\right)$. Recall the process $S^{n, \epsilon}$ given by (4.4). It is easy to check that

$$
\max _{k=0, \ldots, K}\left|S_{\tau_{k}^{n, \epsilon}}^{n, \epsilon}-\Upsilon_{k}\right| \leq \sum_{k=0}^{K-1}\left|\mathbb{E}_{\mathbb{P}_{n}}\left(S_{\left[n \tau_{k+1}^{n, \epsilon}\right]}^{n}-S_{\left[n \tau_{k}^{n, \epsilon}\right]}^{n} \mid \mathcal{F}_{k}^{n, \epsilon}\right)\right|
$$

and $\max _{k=0, \ldots, K}\left|\theta_{k}-\tau_{k}^{n, \epsilon}\right| \leq \lambda$. Therefore, on the event $\left\{\tau_{K}^{n, \epsilon}=1\right\}$ we can estimate the Skorohod distance (on the space $D[0,1]$ )

$$
d\left(S^{n, \epsilon}, D_{(1+\lambda) . .}\right) \leq \lambda+\sum_{k=0}^{K-1}\left|\mathbb{E}_{\mathbb{P}_{n}}\left(S_{\left[n \tau_{k+1}^{n, \epsilon}\right]}^{n}-S_{\left[n \tau_{k}^{n, \epsilon}\right]}^{n} \mid \mathcal{F}_{k}^{n, \epsilon}\right)\right| .
$$

This together with (4.21) and the fact that $H$ is nonnegative and Lipschitz continuous in the Skorohod metric yields that for sufficiently large $n$

$$
\mathbb{E}_{\mathbb{P}_{n}}\left(\frac{Z^{n, \epsilon, K}}{1-\lambda}\right)<\mathbb{E}_{\mathbb{P}_{n}}\left(\frac{H_{1+\lambda}(D)}{1-\lambda}\right)+O(\lambda) .
$$

Next, we treat the term $\sum_{k=0}^{K-1}\left(S_{\left[n \tau_{k+1}^{n, \epsilon}\right]}^{n}-S_{\left[n \tau_{k}^{n, \epsilon}\right]}^{n}\right)^{2}$. From (4.7), on the event $\left\{\tau_{K}^{n, \epsilon}<1\right\}$ we have (recall that $c(\lambda)>2$ )

$$
\sum_{k=0}^{K-1}\left(S_{\left[n \tau_{k+1}^{n, \epsilon}\right]}^{n}-S_{\left[n \tau_{k}^{n, \epsilon]}\right]}^{n}\right)^{2} \geq K \epsilon^{2}-1 \geq \frac{1}{\lambda^{2}} .
$$

On the other hand, on the event $\left\{\tau_{K}^{n, \epsilon}=1\right\}$ we have $\theta_{K}=1+\lambda$ and so, on this event $[D]_{1+\lambda}=\sum_{i=1}^{K}\left(\Upsilon_{i}-\Upsilon_{i-1}\right)^{2}$. From (4.21)-(4.22) we conclude that for sufficiently large $n$

$$
\mathbb{E}\left(\sum_{k=0}^{K-1}\left(S_{\left[n \tau_{k+1}^{n, \epsilon}\right]}^{n}-S_{\left[n \tau_{k}^{n, \epsilon}\right]}^{n}\right)^{2}\right)>\mathbb{E}_{\mathbb{P} D}\left(\frac{1}{\lambda^{2}} \wedge[D]_{1+\lambda}\right)-\epsilon
$$

We arrive to the final step in the estimation $\inf _{\psi \in \mathcal{A}} \Gamma\left(\mathbb{P}_{n}, \psi\right)$. Fix $k=0,1, \ldots, K-1$ and set

$$
\Phi_{k}:=\max \left(\underline{\sigma}^{2}, \frac{\mathbb{E}_{\mathbb{P}_{n}}\left(\left[S^{n}\right]_{\left[n \tau_{k+1}^{n, \epsilon}\right]}-\left[S^{n}\right]_{\left[n \tau_{k}^{n, \epsilon}\right]} \mid \mathcal{F}_{k}^{n, \epsilon}\right)}{\mathbb{E}_{\mathbb{P}_{n}}\left(\frac{\epsilon}{K}+\tau_{k+1}^{n, \epsilon}-\tau_{k}^{n, \epsilon} \mid \mathcal{F}_{k}^{n, \epsilon}\right)}\right) .
$$

From the inequality $\left|X_{i}\right| \geq \underline{\sigma}, i \in \mathbb{N}$, it follows that for sufficiently large $n$ we have $0 \leq \Phi_{k} \mathbb{E}_{\mathbb{P}_{n}}\left(\frac{\epsilon}{K}+\tau_{k+1}^{n, \epsilon}-\tau_{k}^{n, \epsilon} \mid \mathcal{F}_{k}^{n, \epsilon}\right)-\mathbb{E}_{\mathbb{P}_{n}}\left(\left[S^{n}\right]_{\left[n \tau_{k+1}^{n, \epsilon}\right]}-\left[S^{n}\right]_{\left[n \tau_{k}^{n, \epsilon}\right]} \mid \mathcal{F}_{k}^{n, \epsilon}\right) \leq O\left(\frac{\epsilon}{K}\right)$ 
Hence,

$$
\begin{gathered}
\max _{\psi \in \mathcal{A}} \mathbb{E}_{\mathbb{P}_{n}}\left(\frac{\psi_{k}}{2}\left(S_{\left[n \tau_{k+1}^{n, \epsilon}\right]}^{n}-S_{\left[n \tau_{k}^{n, \epsilon]}\right.}^{n}\right)^{2}-\left(\frac{\psi_{k}}{2}+\Lambda \psi_{k}^{2}\right)\left(\left[S^{n}\right]_{\left[n \tau_{k+1}^{n, \epsilon}\right]}-\left[S^{n}\right]_{\left[n \tau_{k}^{n, \epsilon}\right]}\right)\right) \\
\geq O\left(\frac{\epsilon}{K}\right) \min _{\beta \in \mathbb{R}}\left(\frac{\beta}{2}+\Lambda \beta^{2}\right) \\
+\max _{\psi \in \mathcal{A}} \mathbb{E}_{\mathbb{P}_{n}}\left(\frac{\psi_{k}}{2} \mathbb{E}_{\mathbb{P}_{n}}\left(\left(S_{\left[n \tau_{k+1}^{n, \epsilon}\right]}^{n}-S_{\left[n \tau_{k}^{n, \epsilon}\right]}^{n}\right)^{2} \mid \mathcal{F}_{k}^{n, \epsilon}\right)\right. \\
\left.-\Phi_{k} \mathbb{E}_{\mathbb{P}_{n}}\left(\frac{\epsilon}{K}+\tau_{k+1}^{n, \epsilon}-\tau_{k}^{n, \epsilon} \mid \mathcal{F}_{k}^{n, \epsilon}\right)\left(\frac{\psi_{k}}{2}+\Lambda \psi_{k}^{2}\right)\right)
\end{gathered}
$$

The above expression is a quadratic pattern in $\psi_{k}$ which take maximum for

$$
\psi_{k}^{*}=\frac{\mathbb{E}_{\mathbb{P}_{n}}\left(\left(S_{\left[n \tau_{k+1}^{n, \epsilon]}\right.}^{n}-S_{\left[n \tau_{k}^{n, \epsilon]}\right.}^{n}\right)^{2} \mid \mathcal{F}_{k}^{n, \epsilon}\right)}{4 \Lambda \Phi_{k} \mathbb{E}_{\mathbb{P}_{n}}\left(\frac{\epsilon}{K}+\tau_{k+1}^{n, \epsilon}-\tau_{k}^{n, \epsilon} \mid \mathcal{F}_{k}^{n, \epsilon}\right)}-\frac{1}{4 \Lambda} .
$$

Clearly, for sufficiently large $n,\left|\psi_{k}^{*}\right| \leq \ln n$.

Recall the definition of $\zeta^{D}$ given in (4.18). We obtain,

$$
\begin{aligned}
& \max _{\psi \in \mathcal{A}} \sum_{k=0}^{K-1} \mathbb{E}_{\mathbb{P}_{n}}\left(\frac{\psi_{k}}{2}\left(S_{\left[n \tau_{k+1}^{n, \epsilon}\right]}^{n}-S_{\left[n \tau_{k}^{n, \epsilon]}\right.}^{n}\right)^{2}\right. \\
& \left.-\left(\frac{\psi_{k}}{2}+\Lambda \psi_{k}^{2}\right)\left(\left[S^{n}\right]_{\left[n \tau_{k+1}^{n, \epsilon}\right]}-\left[S^{n}\right]_{\left[n \tau_{k}^{n, \epsilon}\right]}\right)\right) \\
& \geq \sum_{k=0}^{K-1} \mathbb{E}_{\mathbb{P}_{n}}\left(\frac{\left(\mathbb{E}_{\mathbb{P}_{n}}\left(\left(S_{\left[n \tau_{k+1}^{n, \epsilon}\right]}^{n}-S_{\left[n r_{k}^{n, \epsilon]}\right.}^{n}\right)^{2} \mid \mathcal{F}_{k}^{n, \epsilon}\right)-\Phi_{k} \mathbb{E}_{\mathbb{P}_{n}}\left(\frac{\epsilon}{K}+\tau_{k+1}^{n, \epsilon}-\tau_{k}^{n, \epsilon} \mid \mathcal{F}_{k}^{n, \epsilon}\right)\right)^{2}}{16 \Lambda \Phi_{k} \mathbb{E}_{\mathbb{P}_{n}}\left(\frac{\epsilon}{K}+\tau_{k+1}^{n, \epsilon}-\tau_{k}^{n, \epsilon} \mid \mathcal{F}_{k}^{n, \epsilon}\right)}\right) \\
& -O(\epsilon) \\
& \geq \frac{1}{16 \Lambda} \sum_{k=0}^{K-1} \mathbb{E}_{\mathbb{P}_{n}}\left(G\left(\frac{\mathbb{E}_{\mathbb{P}_{n}}\left(\left(S_{\left[n \tau_{k+1}^{n, \epsilon}\right]}^{n}-S_{\left[n \tau_{k}^{n, \epsilon]}\right.}^{n}\right)^{2} \mid \mathcal{F}_{k}^{n, \epsilon}\right)}{\mathbb{E}_{\mathbb{P}_{n}}\left(\frac{\epsilon}{K}+\tau_{k+1}^{n, \epsilon}-\tau_{k}^{n, \epsilon} \mid \mathcal{F}_{k}^{n, \epsilon}\right)}\right)\left(\frac{\epsilon}{K}+\tau_{k+1}^{n, \epsilon}-\tau_{k}^{n, \epsilon}\right)\right) \\
& -O(\epsilon) \\
& \geq \frac{1}{16 \Lambda} \sum_{k=0}^{K-1} \mathbb{E}_{\mathbb{P}_{n}}\left(G\left(\zeta_{\theta_{k}}^{D}\right)\left(\theta_{k+1}-\theta_{k}\right)\right)-O(\epsilon) \\
& =\frac{1}{16 \Lambda} \mathbb{E}_{\mathbb{P}_{n}}\left(\int_{0}^{1+\lambda} G\left(\zeta_{t}^{D}\right) d t\right)-O(\epsilon) .
\end{aligned}
$$

The first inequality follows from the above analysis. The second inequality follows from the fact that for sufficiently large $n, \Phi_{k} \in\left[\underline{\sigma}^{2}, \bar{\sigma}^{2}\right], k=0,1, \ldots, K-1$ and the relation $G(z)=\min _{y \in\left[\underline{\sigma}^{2}, \bar{\sigma}^{2}\right]} \frac{(z-y)^{2}}{y}, z \in \mathbb{R}$. We also used the law of total expectation. The third inequality is due to (4.21)-(4.22) and the fact that $\Upsilon_{k}, S_{\left[n \tau_{k}^{n, \epsilon}\right]}^{n}$, $k=0,1 \ldots, K$ are uniformly bounded (in $n$ ). The last equality is trivial.

The proof follows from combining (4.20), and (4.23)-(4.25).

In view of Lemma 4.2 and Proposition 4.6, in order to prove (4.3) (and complete the proof of Proposition 2.4) it remains to establish the following limit theorem. 


\section{Lemma 4.7.}

$$
\begin{aligned}
\underline{\lim }_{\lambda \rightarrow 0} \underline{\lim }_{\epsilon \rightarrow 0} \sup _{D \in \mathcal{D}^{\epsilon, \lambda}} \mathbb{E}_{\mathbb{P}^{D}}\left(\frac{H_{1+\lambda}(D)}{1-\lambda}-\frac{N}{4 \Lambda}\left(\frac{1}{\lambda^{2}} \wedge[D]_{1+\lambda}\right)-\frac{1}{16 \Lambda} \int_{0}^{1+\lambda} G\left(\zeta_{t}^{D}\right) d t\right) \\
\quad \leq \sup _{\nu \in \Gamma} \mathbb{E}_{\mathbb{P} W}\left(H\left(S^{\nu}\right)-\frac{N}{4 \Lambda}\left|S_{1}^{\nu}-s\right|^{2}-\frac{1}{16 \Lambda} \int_{0}^{1} G\left(\nu_{t}^{2}\right) d t\right) .
\end{aligned}
$$

Proof. Fix $\lambda>0$. For any $m \in \mathbb{N}$ let $\epsilon_{m}:=\frac{1}{m}$ and choose $D^{m} \in \mathcal{D}^{\epsilon_{m}, \lambda}$ such that

$$
\begin{aligned}
& \frac{1}{m}+\mathbb{E}_{\mathbb{P}^{D^{m}}}\left(\frac{H_{1+\lambda}\left(D^{m}\right)}{1-\lambda}-\frac{N}{4 \Lambda}\left(\frac{1}{\lambda^{2}} \wedge[D]_{1+\lambda}\right)-\frac{1}{16 \Lambda} \int_{0}^{1+\lambda} G\left(\zeta_{t}^{D^{m}}\right) d t\right) \\
\geq & \sup _{D \in \mathcal{D}^{\epsilon}, \lambda} \mathbb{E}_{\mathbb{P}^{D}}\left(\frac{H_{1+\lambda}(D)}{1-\lambda}-\frac{N}{4 \Lambda}\left(\frac{1}{\lambda^{2}} \wedge[D]_{1+\lambda}\right)-\frac{1}{16 \Lambda} \int_{0}^{1+\lambda} G\left(\zeta_{t}^{D}\right) d t\right) .
\end{aligned}
$$

Following the same arguments as in Lemma 3.9 in [6] gives that there exists a subsequence (which still denoted by $m$ ) and a continuous martingale $M^{\lambda}=\left\{M_{t}^{\lambda}\right\}_{t=0}^{1+\lambda}$ such that we have the weak convergence

$$
\left(D^{m},\left[D^{m}\right], \int_{0}^{\cdot} \zeta_{t}^{D^{m}} d t\right) \Rightarrow\left(M^{\lambda},\left\langle M^{\lambda}\right\rangle,\left\langle M^{\lambda}\right\rangle\right) \quad \text { on } D^{3}[0,1+\lambda] .
$$

As usual $\left\langle M^{\lambda}\right\rangle$ denotes the quadratic variation of the continuous martingale $M^{\lambda}$. From the Skorohod representation theorem (see [14]) it follows that we can construct a probability space (the corresponding probability measure will be denoted by $\mathbb{P}$ ) such that

$$
\left(D^{m},\left[D^{m}\right], \int_{0}^{\cdot} \zeta_{t}^{D^{m}} d t\right) \rightarrow\left(M^{\lambda},\left\langle M^{\lambda}\right\rangle,\left\langle M^{\lambda}\right\rangle\right) \quad \mathbb{P} \text { a.s. }
$$

From (4.15)-(4.18) it follows that

$$
\sup _{m \in \mathbb{N}} \sup _{0 \leq t \leq 1+\lambda} \zeta_{t}^{D^{m}}<\bar{\sigma}^{2}+\sup _{m \in \mathbb{N}} \frac{4 K(1 / m, \lambda)}{\lambda m^{2}}<\infty .
$$

Thus, by Lemma A1.1 in [17] we construct a sequence $\alpha^{m} \in \operatorname{conv}\left(\zeta^{D^{m}}, \zeta^{D^{m+1}}, \ldots\right)$, $m \in \mathbb{N}$ such that $\alpha^{m}$ converge $\mathbb{P} \otimes d t$ almost surely to a stochastic process $\alpha$. From (4.26) - (4.27) and the bounded convergence theorem it follows that

$$
\int_{0}^{t} \alpha_{u} d u=\lim _{m \rightarrow \infty} \int_{0}^{t} \alpha_{u}^{m} d u=\lim _{m \rightarrow \infty} \int_{0}^{t} \zeta_{u}^{D^{m}} d u=\left\langle M^{\lambda}\right\rangle_{t}, \quad \mathbb{P} \otimes d t \text { a.s. }
$$

We conclude that $\alpha=\frac{d\left\langle M^{\lambda}\right\rangle}{d t} \mathbb{P} \otimes d t$ a.s.

From the Fatou lemma and the convexity of $G$ we obtain

$$
\begin{gathered}
\mathbb{E}_{\mathbb{P}}\left(\int_{0}^{1+\lambda} G\left(\frac{d\left\langle M^{\lambda}\right\rangle}{d t}\right) d t\right) \\
\leq \liminf _{m \rightarrow \infty} \mathbb{E}_{\mathbb{P}}\left(\int_{0}^{1+\lambda} G\left(\alpha_{t}^{m}\right) d t\right) \\
\leq \liminf \\
m \rightarrow \infty \\
\mathbb{E}_{\mathbb{P}^{D^{m}}}\left(\int_{0}^{1+\lambda} G\left(\zeta_{t}^{D^{m}}\right) d t\right) .
\end{gathered}
$$

From the dominated convergence theorem and (4.26)

$$
\lim _{m \rightarrow \infty} \mathbb{E}_{\mathbb{P}^{D^{m}}}\left(\frac{1}{\lambda^{2}} \wedge\left[D^{m}\right]_{1+\lambda}\right)=\mathbb{E}_{\mathbb{P}}\left(\frac{1}{\lambda^{2}} \wedge\langle M\rangle_{1+\lambda}\right) .
$$

From the Doob-Kolmogorov inequality, the linear growth of $H$ and (4.27) we conclude that the random variables $\left\{H_{1+\lambda}\left(D^{m}\right)\right\}_{m \in \mathbb{N}}$ are uniformly integrable. This together with (4.26) gives

$$
\lim _{m \rightarrow \infty} \mathbb{E}_{\mathbb{P}^{m}}\left(H_{1+\lambda}\left(D^{m}\right)\right)=\mathbb{E}_{\mathbb{P}}\left(H_{1+\lambda}\left(M^{\lambda}\right)\right) .
$$


By combining (4.28)-4.30 we arrive to

$$
\begin{gathered}
\varliminf_{\lambda \rightarrow 0} \underline{\lim }_{\epsilon \rightarrow 0} \sup _{D \in \mathcal{D} \epsilon, \lambda} \mathbb{E}_{\mathbb{P} D}\left(\frac{H_{1+\lambda}(D)}{1-\lambda}-\frac{N}{4 \Lambda}\left(\frac{1}{\lambda^{2}} \wedge\left[D^{m}\right]_{1+\lambda}\right)-\frac{1}{16 \Lambda} \int_{0}^{1+\lambda} G\left(\zeta_{t}^{D}\right) d t\right) \\
\leq \varliminf_{\lambda \rightarrow 0} \mathbb{E}_{\mathbb{P} D}\left(\frac{H_{1+\lambda}\left(M^{\lambda}\right)}{1-\lambda}-\frac{N}{4 \Lambda}\left(\frac{1}{\lambda^{2}} \wedge\left\langle M^{\lambda}\right\rangle_{1+\lambda}\right)-\frac{1}{16 \Lambda} \int_{0}^{1+\lambda} G\left(\frac{d\left\langle M^{\lambda}\right\rangle}{d t}\right) d t\right) .
\end{gathered}
$$

Finally, it remains to establish the inequality

$$
\begin{gathered}
{\underline{\lim _{\lambda \rightarrow 0}}}_{\lambda \rightarrow \mathbb{E}_{\mathbb{P} D}}\left(\frac{H_{1+\lambda}\left(M^{\lambda}\right)}{1-\lambda}-\frac{N}{4 \Lambda}\left(\frac{1}{\lambda^{2}} \wedge\left\langle M^{\lambda}\right\rangle_{1+\lambda}\right)-\frac{1}{16 \Lambda} \int_{0}^{1+\lambda} G\left(\frac{d\left\langle M^{\lambda}\right\rangle}{d t}\right) d t\right) \\
\leq \sup _{\nu \in \Gamma} \mathbb{E}_{\mathbb{P} W}\left(H\left(S^{\nu}\right)-\frac{N}{4 \Lambda}\left|S_{1}^{\nu}-s\right|^{2}-\frac{1}{16 \Lambda} \int_{0}^{1} G\left(\nu_{t}^{2}\right) d t\right) .
\end{gathered}
$$

Clearly, the right hand side of (4.31) is bigger than $-\frac{\sigma^{2}}{16 \Lambda}$ (just take $\nu \equiv 0$ ). Thus, without loss of generality we can assume that for sufficiently small $\lambda>0$ we have

$$
\mathbb{E}_{\mathbb{P}}\left(\frac{H_{1+\lambda}\left(M^{\lambda}\right)}{1-\lambda}-\frac{N}{4 \Lambda}\left(\frac{1}{\lambda^{2}} \wedge\left\langle M^{\lambda}\right\rangle_{1+\lambda}\right)-\frac{1}{16 \Lambda} \int_{0}^{1+\lambda} G\left(\frac{d\left\langle M^{\lambda}\right\rangle}{d t}\right) d t\right) \geq-\frac{\underline{\sigma}^{2}}{8 \Lambda} .
$$

Otherwise, (4.31) is trivial. From the Burkholder-David-Gundy inequality and the linear Growth of $H$ it follows that there exists a constant $C_{1}>0$ such that

$$
\mathbb{E}_{\mathbb{P}}\left(\frac{H_{1+\lambda}\left(M^{\lambda}\right)}{1-\lambda}\right) \leq C_{1}\left(1+\left\langle M^{\lambda}\right\rangle_{1+\lambda}\right) .
$$

On the other hand it is straight forward to see that there exists a constant $C_{2}>0$ such that $G(z) \geq \frac{z^{2}}{C_{2}}-1, \forall z \geq 0$. From (4.32) we conclude that

$$
\limsup _{\lambda \rightarrow 0} \mathbb{E}_{\mathbb{P}}\left(\int_{0}^{1+\lambda}\left(\frac{d\left\langle M^{\lambda}\right\rangle_{t}}{d t}\right)^{2} d t\right)<\infty .
$$

Finally, for any $\lambda \in(0,1)$ define the continuous martingale $\bar{M}^{\lambda}$ on the time interval $[0,1]$ by $\bar{M}_{t}^{\lambda}:=M_{(1+\lambda) t}^{\lambda}, t \in[0,1]$. Observe that $\bar{M}_{1}^{\lambda}=M_{1+\lambda}^{\lambda}$. From the CauchySchwarz inequality, the Itô Isometry and (4.33) we obtain

$$
\begin{aligned}
& \liminf \operatorname{in}_{\lambda \rightarrow 0} \mathbb{E}_{\mathbb{P}}\left(\frac{H_{1+\lambda}\left(M^{\lambda}\right)}{1-\lambda}-\frac{N}{4 \Lambda}\left(\frac{1}{\lambda^{2}} \wedge\left\langle M^{\lambda}\right\rangle_{1+\lambda}\right)-\frac{1}{16 \Lambda} \int_{0}^{1+\lambda} G\left(\frac{d\left\langle M^{\lambda}\right\rangle}{d t}\right) d t\right) \\
& =\liminf _{\lambda \rightarrow 0} \mathbb{E}_{\mathbb{P}}\left(H_{1+\lambda}\left(\bar{M}^{\lambda}\right)-\frac{N}{4 \Lambda}\left|\bar{M}_{1}^{\lambda}-s\right|^{2}-\frac{1}{16 \Lambda} \int_{0}^{1} G\left(\frac{d\left\langle\bar{M}^{\lambda}\right\rangle}{d t}\right) d t\right) \\
& \leq \sup _{\nu \in \Gamma} \mathbb{E}_{\mathbb{P} W}\left(H\left(S^{\nu}\right)-\frac{N}{4 \Lambda}\left|S_{1}^{\nu}-s\right|^{2}-\frac{1}{16 \Lambda} \int_{0}^{1} G\left(\nu_{t}^{2}\right) d t\right)
\end{aligned}
$$

where the last inequality follows by using the same arguments (based on the randomization technique) as in Lemma 7.2 in [18. This completes the proof.

Remark 4.8. We finish with claiming that the upper bound holds for payoffs of the from given by 2.4). Clearly, Theorem 2.5 remains true for claims which are bounded from below and Lipschitz continuous with respect to the Skorohod metric. Thus, for any $m \in \mathbb{N}$ we have

$$
\begin{gathered}
\lim _{n \rightarrow \infty} \pi_{n}\left(\hat{H}_{m}\left(\left\{S_{[n t]}^{n}\right\}_{t=0}^{1}\right)\right) \\
=\sup _{\nu \in \Gamma} \mathbb{E}_{\mathbb{P} W}\left(\hat{H}_{m}\left(S^{\nu}\right)-\frac{N}{4 \Lambda}\left|S_{1}^{\nu}-s\right|^{2}-\frac{1}{16 \Lambda} \int_{0}^{1} G\left(\nu_{t}^{2}\right) d t\right)
\end{gathered}
$$

where $\hat{H}_{m}$ is given by

$$
\hat{H}_{m}(p):=H(p)-\alpha\left(m \wedge\left|p_{1}-p_{0}\right|^{2}\right), \quad \forall p \in D[0,1] .
$$


Since $\hat{H}_{m} \geq \hat{H}$ then $\pi_{n}\left(\hat{H}_{m}\left(\left\{S_{[n t]}^{n}\right\}_{t=0}^{1}\right)\right) \geq \pi_{n}\left(\hat{H}\left(\left\{S_{[n t]}^{n}\right\}_{t=0}^{1}\right)\right)$ for all $n, m \in$ $\mathbb{N}$. Thus, by using same arguments as in the proof of the inequality (4.31) above, we obtain

$$
\begin{gathered}
\lim _{n \rightarrow \infty} \pi_{n}\left(\hat{H}\left(\left\{S_{[n t]}^{n}\right\}_{t=0}^{1}\right)\right) \\
\leq \lim _{m \rightarrow \infty} \sup _{\nu \in \Gamma} \mathbb{E}_{\mathbb{P} W}\left(\hat{H}_{m}\left(S^{\nu}\right)-\frac{N}{4 \Lambda}\left|S_{1}^{\nu}-s\right|^{2}-\frac{1}{16 \Lambda} \int_{0}^{1} G\left(\nu_{t}^{2}\right) d t\right) \\
=\sup _{\nu \in \Gamma} \mathbb{E}_{\mathbb{P} W}\left(\hat{H}\left(S^{\nu}\right)-\frac{N}{4 \Lambda}\left|S_{1}^{\nu}-s\right|^{2}-\frac{1}{16 \Lambda} \int_{0}^{1} G\left(\nu_{t}^{2}\right) d t\right)
\end{gathered}
$$

as required.

\section{ACKNOWLEDGMENTS}

This research was supported by the GIF Grant 1489-304.6/2019 and the ISF grant $160 / 17$.

\section{REFERENCES}

[1] J. Amendinger, D. Becherer, and M. Schweizer, A monetary value for initial information in portfolio optimization, Finance and Stochastics, 7, 29-46, (2003).

[2] S. Ankirchner, S. Dereich, and P. Imkeller, The Shannon information of filtrations and the additional logarithmic utility of insiders, Annals of Probability, 34, 743-778, (2006).

[3] J. Amendinger, P. Imkeller, and M. Schweizer Additional logarithmic utility of an insider, Stochastic Processes and their Applications, 75, 263-286, (1998).

[4] K. Back, Insider trading in continuous time, The Review of Financial Studies, 5, 387-409, (1992).

[5] P. Bank and D. Baum, Hedging and portfolio optimization in financial markets with a large trader, Math. Finance 14, 1-18, (2004).

[6] P. Bank and Y. Dolinsky, Scaling Limits for Super-replication with Transient Price Impact, Bernoulli, 26, 2176-2201, (2020).

[7] P. Bank, Y. Dolinsky and S. Gökay, Super-replication with nonlinear transaction costs and volatility uncertainty, Annals of Applied Probability. 26, 1698-1726, (2016).

[8] P. Bank, Y. Dolinsky and A.P. Perkki, Superreplication Prices with Small Transaction Costs in the Multivariate Case, Finance and Stoch. 21, 487-508, (2017).

[9] K. Back and H. Pedersen Long-lived information and intraday patterns, Journal of Financial Market, 1, 385-402.

[10] L. Campi, U. Çetin and A. Danilova, Dynamic Markov bridges motivated by models of insider trading, Stochastic Processes and their Applications, 121, 534-567, (2011).

[11] U. Çetin, R. Jarrow and P. Protter, Liquidity risk and arbitrage pricing theory, Finance and Stoch. 8, 311-341, (2004).

[12] P. Cheridito, M. Kupper and L. Tangpi, Duality formulas for robust pricing and hedging in discrete time, SIAM Journal on Financial Mathematics. 8, 738-765, (2017).

[13] A. Danilova, Stock Market Insider Trading in Continuous Time with Imperfect Dynamic Information, Stochastics, 82, 111-131, (2010).

[14] RM. Dudley, Distances of Probability Measures and Random Variables, Ann. Math. Statist. 39, 1563-1572, (1968)

[15] L. Denis and C. Martini, A Theoretical Framework for the Pricing of Contingent Claims in the Presence of Model Uncertainty, Ann. Appl. Probab. 16, 827-852, (2006).

[16] D. Duffie and P. Protter, From Discrete to Continuous Time Finance: Weak Convergence of the Financial Gain Process, Mathematical Finance. 2, 1-15, (1992).

[17] F. Delbaen and W. Schachermayer, A general version of the fundamental theorem of asset pricing, Math. Annalen. 300, 463-520, (1994).

[18] Y. Dolinsky and H.M. Soner, Duality and Convergence for Binomial Markets with Friction, Finance and Stochastics., 17, 447-475, (2013).

[19] H. Föllmer and D. Kramkov, Optional decomposition under constraint, Probability Theory and Related Fields., 109, 1-25, (1997). 
[20] S. Gökay, A.F. Roch and H.M. Soner, Liquidity Models in Continuous and Discrete Time, Advanced Mathematical Methods for Finance, Springer-Verlag, 333366, (2011).

[21] P. Imkeller, Malliavin's Calculus in Insider Models: Additional Utility and Free Lunches, Mathematical Finance, 13, 153-169, (2003).

[22] A. Neufeld and M. Nutz, Superreplication under Volatility Uncertainty for Measurable Claims, Electronic Journal of Probability, 18, 1-14, (2013).

[23] M. Nutz, H.M. Soner, Superhedging and Dynamic Risk Measures under Volatility Uncertainty, SIAM Journal on Control and Optimization, 50, 2065-2089, (2012).

[24] I. Pikovsky and I. Karatzas, Anticipative portfolio optimization, Adv. in Appl. Probab., 28, 1095-1122, (1996).

[25] D. Possamai, G. Royer, and N. Touzi, On the robust superhedging of measurable claims, Electron. Commun. Probab., 18, 113, (2013).

[26] R. Rebolledo, Central limit theorems for local martingales, Probability Theory and Related Fields, 51, 269-286, (1980).

[27] H. Strasser, Mathematical theory of statistics, volume 7 of de Gruyter Studied in Mathematics, Walter de Gruyter \& Co., Berlin, 1985, Statistical experiments and asymptotic decision theory.

[28] H.M. Soner, N. Touzi, and J. Zhang, Martingale representation theorem for the Gexpectation, Stochastic. Processes. Appl. 2, 265-287, (2011).

DEPARTMENT OF STATISTICS, HEBREW UNIVERSITY OF JERUSALEM, ISRAEL. E.MAIL: YAN.DOLINSKY@MAIL.HUJI.AC.IL

DEPARTMENT OF STATISTICS, HEBREW UNIVERSITY OF JERUSALEM, ISRAEL. E.MAIL: JONATHAN.ZOUARI@MAIL.HUJI.AC.IL 